TRANSACTIONS OF THE

AMERICAN MATHEMATICAL SOCIETY

Volume 355, Number 5 , Pages 1933-1959

S 0002-9947(02)03242-7

Article electronically published on December 18, 2002

\title{
RICCI FLATNESS OF ASYMPTOTICALLY LOCALLY EUCLIDEAN METRICS
}

\author{
LEI NI, YUGUANG SHI, AND LUEN-FAI TAM
}

\begin{abstract}
In this article we study the metric property and the function theory of asymptotically locally Euclidean (ALE) Kähler manifolds. In particular, we prove the Ricci flatness under the assumption that the Ricci curvature of such manifolds is either nonnegative or nonpositive. The result provides a generalization of previous gap type theorems established by Greene and Wu, Mok, Siu and Yau, etc. It can also be thought of as a general positive mass type result. The method also proves the Liouville properties of plurisubharmonic functions on such manifolds. We also give a characterization of Ricci flatness of an ALE Kähler manifold with nonnegative Ricci curvature in terms of the structure of its cone at infinity.
\end{abstract}

\section{§1. INTRODUCTION}

In this paper, we will study the geometry of asymptotically locally Euclidean Kähler manifolds. Following [B-K-N], a complete Riemannian manifold $\left(M^{n}, g\right)$ of real dimension $n$ is said to be asymptotically locally Euclidean (ALE) with group $G$ and of order $\lambda(t)$, where $\lambda(t)$ is a nonnegative function defined on $(0, \infty)$ if there exist a compact set $K$ of $M, R>0$, a finite group $G$ of $O(n)$ acting freely on $\mathbb{R}^{n} \backslash B_{0}(R)$, and a diffeomorphism

$$
\psi:\left(\mathbb{R}^{n} \backslash B_{0}(R)\right) / G \rightarrow M \backslash K
$$

such that if $\phi=\psi \circ \pi: \mathbb{R}^{n} \backslash B_{0}(R) \rightarrow M \backslash K$ and if $h=\sum_{i, j} h_{i j} d x^{i} d x^{j}=\phi^{*}(g)$, where $\pi$ is the projection of $\mathbb{R}^{n} \backslash B_{0}(R)$ onto $\left(\mathbb{R}^{n} \backslash B_{0}(R)\right) / G$, then

$$
\begin{cases}\left|h_{i j}(x)-\delta_{i j}\right| & \leq C \lambda(|x|), \\ \left|\frac{\partial h_{i j}(x)}{\partial x^{k}}\right| & \leq C|x|^{-1} \lambda(|x|)\end{cases}
$$

for some constant $C$. If this is the case, then the Christoffel symbols of $h$ satisfy

$$
\left|\Gamma_{i j}^{k}(x)\right| \leq C|x|^{-1} \lambda(|x|)
$$

for some constant $C$. In this work, we always assume that

$$
\lim _{t \rightarrow \infty} \lambda(t)=0
$$

Received by the editors July 25, 2002.

2000 Mathematics Subject Classification. Primary 32Q15.

The research of the first author was partially supported by NSF grant DMS-0196405 and DMS-0203023, USA.

The research of the second author was partially supported by NSF of China, project 10001001.

The research of the third author was partially supported by Earmarked Grant of Hong Kong \#CUHK4217/99P. 
and

$$
\int_{1}^{\infty} t^{-1} \lambda(t)<\infty
$$

In $\mathrm{B}-\mathrm{K}-\mathrm{N}, \lambda(t)=t^{-\eta}$ for some positive constant $\eta>0$. In this case, we also say that $M$ is ALE of order $\eta$. There are several similar definitions of ALE manifolds; see, for example, [Ba], [J], [Kr], [L-P]. Our definition requires the weakest assumption.

We start with the relations of this work to that of other people. In $\mathrm{M}-\mathrm{S}-\mathrm{Y}$ (see also [Si-Y]), Mok, Siu and Yau proved the following:

Theorem (Mok, Siu, and Yau). Let $M^{m}$ be a complete noncompact Kähler manifold with complex dimension $m \geq 2$.

(i) If $M$ has nonnegative holomorphic bisectional curvature such that the volume of the geodesic ball $B_{o}(r)$ centered at o with radius $r$ is greater than or equal to $C r^{2 m}$ for some $C>0$ for all $r$, and if the scalar curvature $\mathcal{R}$ satisfies $\mathcal{R}(x)=O\left(r^{-2-\epsilon}(x)\right)$ for some $\epsilon>0$, then $M$ is isometrically biholomorphic to $\mathbb{C}^{m}$, provided that either $M$ has nonnegative sectional curvature or $M$ is Stein. Here $r(x)$ is the geodesic distance from $x$ to o.

(ii) If $M$ is simply connected with nonpositive sectional curvature such that the scalar curvature $\mathcal{R}$ satisfies $\mathcal{R}(x)=O\left(r^{-2-\epsilon}(x)\right)$ for some $\epsilon>0$, then $M$ is isometrically biholomorphic to $\mathbb{C}^{m}$.

Later, Greene and $\mathrm{Wu}$ G-W 3 generalized the above gap theorem to Riemannian manifolds. They proved the following:

Theorem (Greene and $\mathrm{Wu}$ ). Let $M^{n}$ be a complete noncompact Riemannian manifold with dimension $n \geq 3$. Suppose that

(a) $M$ has a pole;

(b) $M$ has nonnegative sectional curvature or $M$ has nonpositive sectional curvature;

(c) the scalar curvature $\mathcal{R}$ satisfies $\mathcal{R}(x)=O\left(r^{-2-\epsilon}(x)\right)$ for some $\epsilon>0$; and

(d) for the case that $M$ has nonnegative sectional curvature, the volume of $B_{o}(r)$ is greater than or equal to $C r^{n}$ for some $C>0$ for all $r$.

Then $M$ is isometric to $\mathbb{R}^{n}$.

This theorem is not in the most general form in G-W 3]. Moreover, many results in this direction have been obtained; see, for example, [G-P-Z], [K-S, Ds. See also G-P-Z, Ge for a more detailed history.

Note that the curvature tensor of the manifolds in both theorems of Mok, Siu, and Yau and Greene and Wu decay like $r^{-2-\epsilon}$ with volume growth of $B_{o}(r)$ bounded below by $C r^{n}$ where $n$ is the real dimension of the manifolds. By [B-K-N, Theorem 1.1], such a manifold is ALE of order $\eta$ for some $\eta>0$. Notice also that in Mok, Siu, and Yau's theorem (i), in one case, the assumption on the curvature is weaker. It was only assumed that the holomorphic bisectional curvature is nonnegative, while Greene and Wu's theorem works for both nonnegative or nonpositive sectional curvature. One might wonder whether Mok, Siu, and Yau's theorem (ii) is still true if the condition on sectional curvature is replaced by holomorphic bisectional curvature. In this paper, we give an affirmative answer to this question. Namely, we have the following: 
Corollary 3.2. Let $M$ be a complete noncompact Kähler manifold of complex dimension $m \geq 2$. Assume that

(a) $|\mathcal{R}|(x) \leq C r^{-2-\epsilon}(x)$ for some constant $C>0$ where $\mathcal{R}$ is the scalar curvature;

(b) $V_{o}(r) \geq C r^{2 m}$; and

(c) either $M$ has nonnegative holomorphic bisectional curvature or $M$ has nonpositive holomorphic bisectional curvature with only one end.

Then $M$ is isometrically biholomorphic to $\mathbb{C}^{m}$.

On the other hand, asymptotically flat manifolds have been studied by several people $[\mathrm{L}-\mathrm{P}, \mathrm{Ba}]$, etc. The studies are related to the generalization of the positive mass theorem of Schoen and Yau $[\mathrm{Sc}-\mathrm{Y}]$ to higher dimensions. In particular, it was proved in $\mathrm{Ba}$ that a complete asymptotically flat manifold of dimension $n$ of high enough order in the integral sense and with nonnegative Ricci curvature must be flat. The asymptotically locally Euclidean manifolds differ from the asymptotically flat manifolds by allowing a finite fundamental group at the infinity of the manifold. The presence of the finite group makes both the result and the proof of the above-mentioned positive mass type theorem no longer hold. In fact, many nonflat examples have been constructed in $[\mathrm{E}-\mathrm{H}],[\mathrm{G}-\mathrm{H}], \mathrm{C}],[\mathrm{H}],[\mathrm{Kr}],[\mathrm{J}],[\mathrm{T}-\mathrm{Y}]$. Many of them are ALE Kähler with curvatures that decay faster than quadratic and are Ricci flat. Hence it is interesting to see whether the theorems of Mok, Siu and Yau and Greene and Wu can be generalized to the case of Ricci curvature. Namely, if in the assumptions of their theorems, holomorphic bisectional curvature or sectional curvature are replaced by Ricci curvature, then one would like to see whether the conclusion is still true if flatness is replaced by Ricci flatness, provided the curvature tensor has the same decay rates. It is also an interesting problem to generalize the positive mass type theorem for the asymptotically flat manifold to the asymptotically locally Euclidean manifolds. The following major result of this paper provides such a generalization.

Theorem 3.1. Let $M^{m}$ be a complete ALE Kähler manifold of order $\eta>0, m \geq 2$. Suppose that

(a) either $M$ has nonnegative Ricci curvature or $M$ has nonpositive Ricci curvature; and

(b) there exist $1>\epsilon>0$ and $C>0$ such that for all $r>0$,

$$
\frac{1}{V_{o}(r)} \int_{B_{o}(r)}|\mathcal{R}| \leq C r^{-2-\epsilon}
$$

where $\mathcal{R}$ is the scalar curvature of $M, o \in M$ is a fixed point, $B_{o}(r)$ is the geodesic ball of radius $r$ with center $o$ and $V_{o}(r)$ is the volume of $B_{o}(r)$.

Then $M$ is Ricci flat. If, in addition, $\eta \geq 2$, then (b) can be replaced by

$\left(b^{\prime}\right)$

$$
\int_{0}^{r} \frac{t}{V_{o}(t)} \int_{B_{o}(t)}|\mathcal{R}|=o(\log r)
$$

as $r \rightarrow \infty$, and the same conclusion holds.

In particular, if $M$ is a complete noncompact manifold with faster than quadratic decay curvature tensor, with Euclidean volume growth, and with nonnegative or nonpositive Ricci curvature, then $M$ must be Ricci flat (see Corollary 3.1). Note 
that under the definition of ALE in [J], $[\mathrm{Kr}]$, etc., the assumption (b) or $\left(\mathrm{b}^{\prime}\right)$ is automatic; our results then state that if an ALE Kähler metric has nonnegative or nonpositive Ricci curvature, then it is Ricci flat. In [T-Y], and later [J, Theorem 3.3 , it was proved that if $\mathbb{C}^{m} / G$, for a finite group $G \subset S U(m)$, admits a socalled crepant resolution $(X, \pi)$, then each Kähler class of ALE Kähler metrics on $X$ contains a unique Ricci-flat ALE Kähler metric. Our result then concludes that if a complex manifold $X$ is a resolution of $\mathbb{C}^{m} / G$, then it is a crepant resolution if and only if it admits an ALE Kähler metric with nonnegative Ricci curvature. Moreover, the ALE Ricci flat Kähler metric constructed in [T-Y] and [J] is the only one with nonnegative (or nonpositive) Ricci curvature among each Kähler class. The result in $\mathrm{Yg}$ shows that (b) (or $\left(\mathrm{b}^{\prime}\right)$ ) in Theorem 3.1 is also sharp.

Note that a complex submanifold of $\mathbb{C}^{N}$ is of nonpositive holomorphic bisectional curvature. Using methods similar to the proof of Theorem 3.1, we give an alternative proof of an interesting result of Moore [Me, Theorem 2], which says that if $M^{m}$ is a complete immersed complex submanifold in $\mathbb{C}^{N}$ with complex dimension $m \geq 2$ and with finite total scalar curvature, then $M$ must be an affine complex linear subspace.

Some gap theorems were obtained under the assumption that the fundamental group of the tangent cone at infinity of the manifold is trivial; see [Ge for a description. In our case, if $M^{m}$ is an ALE Kähler manifold, it is not hard to prove that the tangent cone of $M$ has a complex structure and the group $G$ in the definition of ALE manifold is a subgroup of $U(m)$ with respect to this complex structure. If, in addition, $M$ is simply connected and Ricci flat, then $G$ is in fact in $S U(m)$. It turns out that if $M$ has nonnegative Ricci curvature, the condition that $G \subset S U(m)$ is also sufficient for $M$ to be Ricci flat. Hence, in this case, we can replace conditions (b) or $\left(\mathrm{b}^{\prime}\right)$ in Theorem 3.1 above by the condition that $G \subset S U(m)$. In fact, one can relax other conditions also; see Theorem 4.1 for details.

The methods in the proof of Theorem 3.1 can be used to study Liouville properties of plurisubharmonic functions on ALE Kähler manifolds. This kind of problem is closely related to the study of the geometry of the complex manifolds. For example, in the original proof of Mok, Siu and Yau's theorem (i), a bounded plurisubharmonic function $u$ was constructed so that $\sqrt{-1} \partial \bar{\partial} u$ is the Ricci form. Then it was proved that $u$ is actually constant and hence $M$ must be flat. The Liouville type problem that we are interested in is as follows. It is well known that on $\mathbb{C}$, any subharmonic function of sublogarithmic growth is constant. Therefore, the same conclusion holds for plurisubharmonic functions on $\mathbb{C}^{m}$. It will be interesting to see under what conditions the same result still holds for Kähler manifolds. In $[\mathrm{N}]$, the first author proved that if $M$ can be compactified, then any bounded plurisubharmonic function on $M$ is a constant. In this paper, we shall prove similar Liouville theorems for plurisubharmonic functions on complete ALE Kähler manifolds. For example, we show that on a complete ALE Kähler manifold of order $\eta \geq 2$, any plurisubharmonic function of sublogarithmic growth must be a constant. Although it seems natural to study plurisubharmonic functions on complete Kähler manifolds, to the authors's knowledge, very little is known. It is conjectured that a similar Liouville theorem holds on complete Kähler manifolds with nonnegative Ricci curvature.

We hope that our results will be useful in classifying ALE Kähler manifolds, which so far has only been done, up to diffeomorphism, for hyper-Kähler surfaces 
in $[\mathrm{Kr}$. On the other hand, even if one could have a classification for all the ALE Kähler manifolds, it is unlikely that the proofs to Theorems 3.1 and 4.1 could be made any easier. It is an open question whether this result on Ricci flatness remains true in the category of Riemannian spin manifolds. This will be an interesting issue to investigate in the future.

The paper is organized as follows. In $\S 2$, we construct plurisubharmonic functions which will be used later. In §3, we study Ricci flatness of ALE Käher metrics in terms of curvature decays. In $\S 4$, we study Ricci flatness of ALE Kähler manifolds in terms of the group $G$ at infinity. In $\S 5$, we will prove various Liouville-type theorems for plurisubharmonic functions on ALE Kähler manifolds.

Acknowledgments. The authors would like to thank Laszlo Lempert, Hing-Sun Luk, Hung-Hsi Wu, Xi Zhang and Kang Zuo for helpful discussions. We would also like to thank Peter Li and Gang Tian for their interest in this work.

\section{§2. Construction of Plurisubharmonic exhaustion functions}

Let $\left(M^{m}, g\right)$ be a complete ALE Kähler manifold of complex dimension $m$ of order $\lambda(t)$ so that (1.1), (1.3) and (1.4) hold. In this section, we always use the same set-up as in the beginning of $\S 1$. We are going to construct plurisubharmonic exhaustion functions with various growth rates depending on $\lambda(t)$.

First, let us begin with the following simple lemma.

Lemma 2.1. With the above notation, suppose $M$ is ALE of order $\lambda$ which satisfies (1.3). Then there exist constants $C, b>0$ such that

(a)

$$
C^{-1} d(\phi(x), K)-b \leq d_{0}\left(x, B_{0}(R)\right) \leq C d(\phi(x), K)+b
$$

where $d_{0}$ is the Euclidean distance;

(b) for any real numbers $c_{i}, d_{i}, 1 \leq i \leq n$,

$$
\left|\sum_{i} c_{i} d_{i}-\sum_{i j} h_{i j}(x) c_{i} d_{j}\right|=\left|\sum_{i, j}\left(\delta_{i j}-h_{i j}\right) c_{i} d_{j}\right| \leq C \lambda(|x|)|c||d|
$$

for some constant $C$, where $|c|=\left(\sum_{i} c_{i}^{2}\right)^{\frac{1}{2}}$ and $|d|$ is defined similarly; and

(c) for any $r$,

$$
C^{-1} r^{n} \leq V_{o}(r) \leq C r^{n}
$$

where $V_{o}(r)$ is the volume of the geodesic ball $B_{o}(r)$ centered at a fixed point $o \in M$ and with radius $r$.

Proof. By (1.1), the assumption that $\lim _{t \rightarrow \infty} \lambda(t)=0$ and the fact that $G$ is finite, the results follow easily.

Let $q(x)=1+\sum_{i=1}^{2 m}\left(x_{i}\right)^{2}$ be defined on $\mathbb{R}^{2 m}$. Since $q$ is invariant under the action of $O(2 m)$, then $q$ descends to be a function on $M \backslash K$, which will also be denoted by $q$.

Lemma 2.2. Suppose $\lambda(t)=t^{-\eta}$ for some $\eta>0$. For any $\alpha>0$, if $f=q^{\alpha}$, then

(a) $f$ is strictly plurisubharmonic outside a compact set and is an exhaustion function in the sense that $\lim _{\tilde{x} \rightarrow \infty} f(\tilde{x})=\infty$; and 
(b) there is a constant $C>0$ such that

$$
|\nabla f|(\tilde{x}) \leq C r^{2 \alpha-1}(\tilde{x})
$$

and

$$
|\operatorname{Hess}(f)(\tilde{x})(X, X)| \leq C r^{2 \alpha-2}(\tilde{x})|X|^{2},
$$

at the point $\tilde{x}$, where $\operatorname{Hess}(f)$ is the Hessian of $f$ and $r(\tilde{x})$ is the distance of $\tilde{x}$ from a fixed point.

Proof. By Lemma 2.1, it is easy to see that $f$ is an exhaustion function. Also, it is sufficient to prove that the lemma is true for the function $f(x)=q^{\alpha}(x)$ on $\mathbb{R}^{2 m} \backslash$ $B_{0}(R)$ with the metric $h$ with $r(\tilde{x})$ replaced by $|x|$. At a point $x=\left(x^{1}, \ldots, x^{2 m}\right)$,

$$
\frac{\partial}{\partial x^{i}} f(x)=2 \alpha x^{i} q^{\alpha-1}(x)
$$

and

$$
\operatorname{Hess}(f)\left(\frac{\partial}{\partial x^{i}}, \frac{\partial}{\partial x^{j}}\right)=2 \alpha q^{\alpha-2}\left[q \delta_{i j}+2(\alpha-1) x^{i} x^{j}-g \Gamma_{i j}^{k} x^{k}\right] .
$$

By (1.1)-(1.3), part (b) of the lemma follows.

By Lemma 1.13 in G-W 1, to prove that $f$ is strictly plurisubharmonic outside a compact set, it is sufficient to prove that at a point $x$ outside some compact set of $\mathbb{R}^{2 m}$, if $X$ and $Y$ are unit vectors with $X \perp Y$ with respect to $h$ in the tangent space of $x$, then

$$
\operatorname{Hess}(f)(X, X)+\operatorname{Hess}(f)(Y, Y)>0 .
$$

Let $X=\sum_{i} a_{i} \frac{\partial}{\partial x^{i}}$ and $Y=\sum_{i} b_{i} \frac{\partial}{\partial x^{i}}$ be two orthonormal tangent vectors at $x$. By (2.3), we have

$$
\begin{aligned}
& \operatorname{Hess}(f)(X, X)+\operatorname{Hess}(f)(Y, Y) \\
& =\sum_{i, j}\left(a_{i} a_{j}+b_{i} b_{j}\right) \operatorname{Hess}(f)\left(\frac{\partial}{\partial x^{i}}, \frac{\partial}{\partial x^{j}}\right) \\
& =2 \alpha q^{\alpha-2} \sum_{i, j}\left(a_{i} a_{j}+b_{i} b_{j}\right)\left[q \delta_{i j}+2(\alpha-1) x^{i} x^{j}-q \Gamma_{i j}^{k} x^{k}\right] \\
& \geq 2 \alpha q^{\alpha-2}\left[q \sum_{i}\left(a_{i}^{2}+b_{i}^{2}\right)+2(\alpha-1)\left(\left(\sum_{i} a_{i} x^{i}\right)^{2}+\left(\sum_{i} b_{i} x^{i}\right)^{2}\right)\right. \\
& \left.\quad-C_{1} q|x|^{-\eta}\right]
\end{aligned}
$$

for some positive constant $C_{1}$. By Lemma 2.1 (b),

$$
\begin{aligned}
\sum_{i}\left(a_{i}^{2}+b_{i}^{2}\right) & \geq \sum_{i j} h_{i j}\left(a_{i} a_{j}+b_{i} b_{j}\right)-C_{3}|x|^{-\eta} \sum_{i}\left(a_{i}^{2}+b_{i}^{2}\right) \\
& =2-C_{3}|x|^{-\eta} \sum_{i}\left(a_{i}^{2}+b_{i}^{2}\right) \\
& \geq 2-C_{3}|x|^{-\eta},
\end{aligned}
$$


for some constant $C_{3}$, and

$$
\begin{aligned}
\left(\sum_{i} a_{i} x^{i}\right)^{2} & \leq\left(\left|\sum_{i, j} h_{i j} a_{i} x^{j}\right|+C_{4}|x|^{-\eta+1}|a|\right)^{2} \\
& \leq\left(\sum_{i, j} h_{i j} a_{i} x^{j}\right)^{2}+C_{5}|x|^{-\eta+2}
\end{aligned}
$$

for some constants $C_{4}, C_{5}$, where we have used the fact that $|a| \leq C|X|=C$ for some constant $C$ independent of $x$ and $X$, where $X$ is a unit vector. Similarly,

$$
\left(\sum_{i} b_{i} x^{i}\right)^{2} \leq\left(\sum_{i, j} h_{i j} b_{i} x^{j}\right)^{2}+C_{6}|x|^{-\eta+2} .
$$

Since $X$ and $Y$ are orthonormal (consider the vector $Z=\sum_{i} x^{i} \frac{\partial}{\partial x^{i}}$ ), we have

$$
\langle X, Z\rangle^{2}+\langle Y, Z\rangle^{2} \leq|Z|^{2}
$$

where the inner products are taken with respect to $h$. Hence,

$$
\begin{aligned}
\left(\sum_{i, j} h_{i j} b_{i} x^{j}\right)^{2}+\left(\sum_{i, j} h_{i j} a_{i} x^{j}\right)^{2} & \leq \sum_{i, j} h_{i j} x^{i} x^{j} \\
& \leq|x|^{2}+C_{7}|x|^{-\eta+2}
\end{aligned}
$$

for some constant $C_{7}$. Combining (2.4)-(2.8), we have

$$
\begin{aligned}
\operatorname{Hess} & (f)(X, X)+H(f)(Y, Y) \\
& \geq 2 \alpha q^{\alpha-2}\left[q\left(2-C_{3}|x|^{-\eta}\right)-2(1-\alpha)\left(|x|^{2}+C_{8}|x|^{-\eta+2}\right)-C_{1} q|x|^{-\eta}\right] \\
& \geq 2 \alpha q^{\alpha-2}\left[2 \alpha|x|^{2}-C_{9}|x|^{-\eta+2}-C_{1} q|x|^{-\eta}\right] \\
\quad & >0
\end{aligned}
$$

if $|x|$ is large. Here $C_{8}$ and $C_{9}$ are positive constants independent of $x$. This completes the proof of the lemma.

If $M$ is ALE of order $\eta \geq 2$, we may get a better exhaustion function. In this case, let $q_{1}(x)=1+\alpha \sum_{i=1}^{2 m}\left(x^{i}\right)^{2}$. Since $q_{1}$ descends to $M \backslash K$, we denote this function by $q_{1}$ again.

Lemma 2.3. Suppose $M^{m}$ is an ALE Kähler manifold of order $\eta \geq 2$. Then there exists $\alpha>0$ such that the function $F(\tilde{x})=\log q_{1}(\tilde{x})$ satisfies

(a) $F$ is strictly plurisubharmonic outside a compact set and is an exhaustion function in the sense that $\lim _{\tilde{x} \rightarrow \infty} F(\tilde{x})=\infty$; and

(b) there is a constant $C>0$ such that

$$
\begin{gathered}
|\nabla F|(\tilde{x}) \leq C r^{-1}(\tilde{x}), \\
|\operatorname{Hess}(F)(\tilde{x})(X, X)| \leq C r^{-2}(\tilde{x})|X|^{2}
\end{gathered}
$$

at the point $\tilde{x}$, where $\operatorname{Hess}(F)$ is the Hessian of $F$ and $r(\tilde{x})$ is the distance of $\tilde{x}$ from a fixed point. 
Proof. It is sufficient to prove the case that $\eta=2$. The proof of (b) is similar to that of Lemma 2.2. It is easy to see that $F$ is an exhaustion function. It remains to prove that $F$ is strictly plurisubharmonic outside a compact set. As in the proof of Lemma 2.2, it is sufficient to prove that $F(x)=\log q_{1}(x)$ is strictly plurisubharmonic outside a compact set in $\mathbb{R}^{2 m} \backslash B_{0}(R)$ if $1>\alpha>0$ is chosen to be small enough. Using the same notation as in the proof of Lemma 2.2, we have

$$
\begin{aligned}
\operatorname{Hess}(F) & (X, X)+\operatorname{Hess}(F)(Y, Y) \\
& =\sum_{i, j}\left(a_{i} a_{j}+b_{i} b_{j}\right) H(F)\left(\frac{\partial}{\partial x^{i}}, \frac{\partial}{\partial x^{j}}\right) \\
& =2 \alpha q_{1}^{-2} \sum_{i, j}\left(a_{i} a_{j}+b_{i} b_{j}\right)\left[q_{1} \delta_{i j}-2 \alpha x^{i} x^{j}-q_{1} \Gamma_{i j}^{k} x^{k}\right] \\
& \geq 2 \alpha q_{1}^{-2}\left[q_{1}\left(2-C_{1}|x|^{-2}\right)-2 \alpha\left(|x|^{2}+C_{2}\right)-C_{3} q_{1}|x|^{-2}\right] \\
& \geq 2 \alpha q_{1}^{-2}\left[2-C_{4} q_{1}|x|^{-2}-2 \alpha C_{2}\right]
\end{aligned}
$$

for some positive constants $C_{1}-C_{4}$ independent of $\alpha$. Hence, if $\alpha>0$ is small enough, $F$ will be strictly plurisubharmonic outside a compact set.

If $M^{m}$ is ALE of order $\lambda(t)$ which only satisfies (1.3), we still can construct a smooth exhaustion function that is strictly plurisubharmonic outside a compact set.

Lemma 2.4. Let $M^{m}$ be a complete noncompact Kähler manifold which is ALE of order $\lambda(t)$, where $\lambda$ satisfies (1.3). Let $q$ be the function as in Lemma 2.2. Then the exhaustion function $q$ is strictly a plurisubharmonic function outside a compact set.

Proof. The proof is similar to the proof of Lemma 2.2. We simply let $\alpha=1$ there and replace $|x|^{-\eta}$ by $\lambda(|x|)$. It is easy to see that the lemma is true.

Next, we want to extend the strictly plurisubharmonic functions constructed in the previous lemmas to plurisubharmonic functions on $M$.

Lemma 2.5. Let $M^{m}$ be a complete noncompact Kähler manifold such that there exists a smooth exhaustion function $f$ that is strictly plurisubharmonic outside a compact set. Then there is a smooth plurisubharmonic function $h$ on $M$ such that $h \equiv \lambda(f+C)$ outside a compact set for some positive constant $\lambda$ and constant $C$. Moreover, $h$ is strictly plurisubharmonic except possibly at finitely many compact nontrivial subvarieties.

Proof. First, note that there is an integer $k$ such that any nontrivial compact subvariety of positive dimension is contained in a fixed compact set which is a subset of some sublevel set $\{f<k\}$ by the maximum principle. We may assume that $k=0$. Moreover, by multiplying $f$ by a positive constant, without loss of generality, we may assume that $f$ is strictly plurisubharmonic outside $\{f<-1\}$, which we may also assume to be nonempty and to have compact closure in $\{f<0\}$. We may also assume that there exist positive integers $k_{2}>k_{1}>10$ such that

$$
\Omega_{0}=\{f<0\} \Subset \Omega_{1}=\left\{f<k_{1}\right\} \Subset \Omega_{2}=\left\{f<k_{2}\right\} \Subset M .
$$

Since every point of $\partial \Omega_{2}$ is strictly pseudo-convex, by Gt] (see Theorem 4 in G-R. p. 273]), there is a Stein space $Y$, a holomorphic map $\pi: \Omega_{2} \rightarrow Y$, and there is 
a finite set (which may be empty) of points $y_{1}, \ldots, y_{k}$ in $Y$ such that $\pi^{-1}\left(y_{i}\right)$ is a compact subvariety of $\Omega_{2}$ of positive dimension, and $\pi: \Omega_{2} \backslash \bigcup_{i=1}^{k} \pi^{-1}\left(y_{i}\right) \rightarrow$ $Y \backslash\left\{y_{1}, \ldots, y_{k}\right\}$ is biholomorphic. On the other hand, by the lemma in $\S 3$ in $\mathrm{Na}$, there is a real analytic strictly plurisubharmonic exhaustion function $\psi$ on $Y$. Hence, $\psi \circ \pi$ is a $C^{2}$ function on $\Omega_{2}$ which is strictly plurisubharmonic outside $\bigcup_{i=1}^{k} \pi^{-1}\left(y_{i}\right)$. Then we have a smooth plurisubharmonic exhaustion function $\phi=\psi \circ \pi$ on $\Omega_{2}$, which is strictly plurisubharmonic except possibly at finitely many nontrivial subvarieties of positive dimension. In particular, $\phi$ is strictly plurisubharmonic on $\Omega_{2} \backslash \Omega_{0}$. We may assume that $\phi>0$ on $\bar{\Omega}_{1}$. Let $\lambda=\max _{\Omega_{1}} \phi$, which is positive. Define

$$
g=\left\{\begin{array}{l}
\max \{\lambda f, \phi\}, \text { on } \Omega_{1}, \\
\lambda f, \text { outside } \Omega_{1} .
\end{array}\right.
$$

In $\Omega_{0}, g=\phi$. Near $\partial \Omega_{1}, \lambda f \geq \frac{1}{2} k_{1} \lambda>5 \lambda>\phi$ and $g=\lambda f$ there. In $\Omega_{1} \backslash \Omega_{0}$, $g=\max \{\lambda f, \phi\}$. Hence, $g$ is locally Lipschitz continuous, strictly plurisubharmonic (in the weak sense) except possible outside the union $L$ of finitely many nontrivial subvarieties with $L$ being contained in $\Omega_{0}$. Moreover, $g$ is smooth in $\Omega_{0}$ and outside $\Omega_{1}$.

Let $K=\bar{\Omega}_{1} \backslash \Omega_{0}$ and let $V, W$ be open sets with $K \Subset V \Subset W \Subset \Omega_{2}$ and $\bar{W} \cap L=\emptyset$. Note that $g$ is smooth outside $K$. We claim that for any $\epsilon>0$, there is a smooth function $g_{\epsilon}$ defined on a neighborhood of $\bar{W}$ such that $g_{\epsilon}$ is strictly plurisubharmonic on $W$ and

$$
\left|g-g_{\epsilon}\right|+\left|\nabla g-\nabla g_{\epsilon}\right|+\left|\nabla^{2} g-\nabla^{2} g_{\epsilon}\right| \leq \epsilon
$$

on $\bar{W} \backslash V$.

Suppose the claim is true. Let $\psi \geq 0$ be a smooth function such that $\psi=1$ in $V$ and $\psi=0$ outside $\bar{W}$. Let $h=(1-\psi) g+\psi g_{\epsilon}$. Then $h$ is smooth. $h=g_{\epsilon}$ in $V$ and hence is strictly plurisubharmonic there. $h=g$ in $M \backslash \bar{W}$, which is a subset of $\Omega_{0} \cup\left(M \backslash \Omega_{1}\right)$. Hence, $h=\phi$ or $h=\lambda f$ and $h$ is also strictly plurisubharmonic there except at $L$. In particular, $h=\lambda f$ outside $\Omega_{2}$. It remains to consider the points in $\bar{W} \backslash V$. In this set

$$
h=g+\psi\left(g_{\epsilon}-g\right)
$$

Note that in this set $g$ is smooth, $g=\phi$ or $g=\lambda f$ and $g$ is strictly plurisubharmonic. Since $\psi$ is a fixed cutoff function, it is easy to see that $h$ is strictly plurisubharmonic in $\bar{W} \backslash V$ by (2.11) provided $\epsilon>0$ is small.

We will prove (2.11) in the following lemma, which is essentially proved in section 4 of [G-W 2] For the sake of completeness we include a proof here.

Lemma 2.6. Let $M$ be a complex manifold and $K$ a compact set and let $f$ be a continuous plurisubharmonic function on $M$ such that $f$ is smooth on $M \backslash K$ and $f$ is strictly plurisubharmonic (in a weak sense) on a neighborhood $U$ of $K$. Let $K \subset V \Subset W \Subset U$. For any $\epsilon>0$ there is a smooth strictly plurisubharmonic function $g$ defined on a neighborhood of $\bar{W}$ such that

$$
|f-g|+|\nabla f-\nabla g|+\left|\nabla^{2} f-\nabla^{2} g\right| \leq \epsilon
$$

on $\bar{W} \backslash V$. 
Proof. There exist coordinate neighborhoods $\mathcal{O}_{i}$ and $\mathcal{P}_{i}, 1 \leq i \leq k$ such that $\mathcal{O}_{i} \subset \mathcal{P}_{i} \Subset U$, and $\mathcal{O}_{i}$ and $\mathcal{P}_{i}$ are diffeomorphic to $\{|x|<1\}$ and $\{|x|<2\}$ respectively. Moreover, $\bar{W} \subset \bigcup_{i} \mathcal{O}_{i}$. First, we prove that given any $\epsilon>0$ there exists a continuous strictly plurisubharmonic function $h_{1}$ defined on $U$ such that $h_{1}$ is smooth on $\mathcal{O}_{1}$ and a neighborhood of $\bar{W} \backslash V$; moreover,

$$
\left|f-h_{1}\right|+\left|\nabla f-\nabla h_{1}\right|+\left|\nabla^{2} f-\nabla^{2} h_{1}\right| \leq \epsilon
$$

on $\bar{W} \backslash V$. Let $\phi$ be a smooth cutoff function such that $\phi \equiv 1$ in the neighborhood of $\bar{W} \backslash V$ and supp $\phi \cap K=\emptyset$. Let $\eta$ be another cutoff function such that $\eta \equiv 1$ on the complement of a neighborhood of $(\bar{W} \backslash V) \cup \overline{\mathcal{O}}_{1}$, and $\eta \equiv 0$ on $(\bar{W} \backslash V) \cup \overline{\mathcal{O}}_{1}$.

Since $f$ is strictly plurisubharmonic on $U$, by convolution (mollifying), there is a function $g_{1}$ defined on $\mathcal{P}_{1}$ such that $g_{1}$ is strictly plurisubharmonic on $\mathcal{P}_{1}$ and

$$
\left|f-g_{1}\right|+\left|\nabla f-\nabla g_{1}\right|+\left|\nabla^{2} f-\nabla^{2} g_{1}\right| \leq \epsilon
$$

on $\mathcal{P}_{1} \cap \bar{W} \backslash V$ and $\left|f-g_{1}\right| \leq \epsilon$ on $\mathcal{P}_{1}$, since $f$ is continuous and is smooth near $\bar{W} \backslash V$. Let $h=\phi f+(1-\phi) g_{1}$. Then $h=f$ near $\bar{W} \backslash V, h=g_{1}$ on $\mathcal{P}_{1} \cap K$, and $h=\phi f+(1-\phi) g_{1}$ on $\mathcal{P}_{1} \backslash K$. Hence, $h$ is defined and smooth on $\mathcal{P}_{1}$ and a neighborhood of $\bar{W} \backslash V$. Moreover, $|f-h| \leq \epsilon$ there and by choosing a better $g_{1}$, we may also have

$$
|f-h|+|\nabla f-\nabla h|+\left|\nabla^{2} f-\nabla^{2} h\right| \leq \epsilon
$$

on $\mathcal{P}_{1} \cap \bar{W} \backslash V$. Now we extend $h$ to a continuous strictly plurisubharmonic function on $U$ in the following way:

$$
h_{1}=\left\{\begin{array}{l}
f \text { on the set where } \eta=1, \\
\max \{f, h+2 \epsilon-4 \epsilon \eta\} \text { on the set } 0<\eta<1, \\
h+2 \epsilon \text { on the set } \eta=0 .
\end{array}\right.
$$

Note that near $\eta=1, h+2 \epsilon-4 \epsilon \eta<h-\epsilon$ and near $\eta=0, h+2 \epsilon-4 \epsilon \eta>h+\epsilon$. Since $|f-h| \leq \epsilon$, near $\eta=1, h_{1}=f$ and near $\eta=0, h_{1}=h+2 \epsilon$. Hence, $h_{1}$ is well defined and continuous. Note that the complex Hessian of $g_{1}$ is bounded below by a fixed positive constant if $\epsilon>0$ is small because $f$ is strictly plurisubharmonic. Hence, the complex Hessian of $h$ is also bounded below by a positive constant. Since $\eta$ is fixed, one can choose $\epsilon>0$ small enough so that $h+2 \epsilon-4 \epsilon \eta$ is strictly plurisubharmonic. Hence, $h_{1}$ is strictly plurisubharmonic on $U$. Since $h_{1}=h+2 \epsilon$ on $(\bar{W} \backslash V) \cup \overline{\mathcal{O}}_{1}, h_{1}$ is smooth there. Now we can replace $\bar{W} \backslash V$ by $\bar{W} \backslash V \cup \overline{\mathcal{O}}_{1}$ and repeat the process to get a continuous strictly plurisubharmonic function on $U$ that is smooth near $\bar{W} \backslash V \cup \overline{\mathcal{O}}_{1} \cup \overline{\mathcal{O}}_{2}$ and that satisfies the condition (2.12) on $\bar{W} \backslash V$. Continuing in this way, we get the function $g$ that we want.

Combining Lemmas $2.2-2.5$, we have the following:

Proposition 2.1. Let $M^{m}$ be a complete ALE Kähler manifold of order $\lambda(t)$ satisfying (1.3).

(a) $M$ supports a smooth plurisubharmonic exhaustion function that is strictly plurisubharmonic except possibly at finitely many nontrivial compact subvarieties of positive dimension.

(b) Suppose $\lambda(t)=t^{-\eta}$ for some constant $\eta>0$. Then for any $\alpha>0$, there exists a smooth plurisubharmonic exhaustion function $u$ on $M$ such that it is strictly plurisubharmonic except possibly at finitely many nontrivial 
compact subvarieties of positive dimension and it satisfies (2.1) and (2.2) in Lemma 2.2.

(c) If, in addition, $\eta \geq 2$, then there exists a smooth plurisubharmonic exhaustion function $u$ on $M$ such that it is strictly plurisubharmonic except possibly at finitely many nontrivial compact subvarieties of positive dimension and it satisfies (2.9) and (2.10) in Lemma 2.3.

Remark 2.1. In our definition, if $M$ is ALE, then it has only one end. However, the arguments also work in the case where $M$ has finitely many ends such that each end is ALE in the obvious sense. In particular, Proposition 2.1 is still true in this case, provided the assumptions are true for each end.

\section{§3. Ricci Flatness of ALE KäHLER MEtrics}

Now we can prove the following main theorem:

Theorem 3.1. Let $M^{m}$ be a complete ALE Kähler manifold of order $\eta>0, m \geq 2$. Suppose that

(a) either $M$ has nonnegative Ricci curvature or $M$ has nonpositive Ricci curvature; and

(b) there exist $1>\epsilon>0$ and $C>0$ such that for all $r>0$,

$$
\frac{1}{V_{o}(r)} \int_{B_{o}(r)}|\mathcal{R}| \leq C r^{-2-\epsilon}
$$

where $\mathcal{R}$ is the scalar curvature of $M, o \in M$ is a fixed point, $B_{o}(r)$ is the geodesic ball of radius $r$ with center o and $V_{o}(r)$ is the volume of $B_{o}(r)$.

Then $M$ is Ricci flat. If, in addition, $\eta \geq 2$, then (b) can be replaced by

$$
\int_{0}^{r} \frac{t}{V_{o}(t)} \int_{B_{o}(t)}|\mathcal{R}|=o(\log r)
$$

as $r \rightarrow \infty$, and the same conclusion holds.

Proof. We only consider the case that $M$ has nonnegative Ricci curvature. The other case is similar. Let $0<\alpha<\epsilon /(2 m-2)$ be a constant. By Proposition 2.1, since $M$ is ALE of order $\eta>0$, there is a smooth plurisubharmonic function $u$ that is strictly plurisubharmonic except possibly at finitely many nontrivial subvarieties $V_{1}, \ldots, V_{k}$ of $M$. Moreover, there exists a constant $C_{1}>0$ such that

$$
|\nabla u|(x) \leq C_{1} r^{2 \alpha-1}(x)
$$

and

$$
\left|\nabla^{2} u\right|(x) \leq C_{1} r^{2 \alpha-2}(x),
$$

where $r(x)$ is the distance from $x \in M$ to $o$. Let $x_{0} \notin \bigcup_{j=1}^{k} V_{j}$ be any point. Then $u$ is strictly plurisubharmonic at $x_{0}$. Suppose $\operatorname{Ric}\left(x_{0}\right) \neq 0$. Then

$$
\int_{B_{x_{0}}(1)}(\sqrt{-1} \partial \bar{\partial} u)^{m-1} \wedge \text { Ric }>0 .
$$


On the other hand, if $R>r\left(x_{0}\right)+1$, using the fact that $u$ is plurisubharmonic and Ric is nonnegative everywhere, we have

$$
\begin{aligned}
\int_{B_{x_{0}}(1)}(\sqrt{-1} \partial \bar{\partial} u)^{m-1} \wedge \operatorname{Ric} & \leq \int_{B_{o}(R)}(\sqrt{-1} \partial \bar{\partial} u)^{m-1} \wedge \text { Ric } \\
& =\int_{\partial B_{o}(R)} \sqrt{-1} \bar{\partial} u \wedge(\sqrt{-1} \partial \bar{\partial} u)^{m-2} \wedge \text { Ric } \\
& \leq C_{2} R^{2 \alpha-1} \cdot R^{(m-2)(2 \alpha-2)} \int_{\partial B_{o}(R)} \mathcal{R}
\end{aligned}
$$

for some constant $C_{3}$ independent of $R$. Here we have used (3.1) and (3.2). By assumption (b) and Lemma 2.1(c), there exist $R_{i} \rightarrow \infty$ such that

$$
\int_{\partial B_{o}\left(R_{i}\right)} \mathcal{R} \leq C_{3} R_{i}^{2 m-3-\epsilon}
$$

for some constant $C_{3}$ independent of $i$. Combining this with (3.4), we have

$$
\int_{B_{x_{0}}(1)}(\sqrt{-1} \partial \bar{\partial} u)^{m-1} \wedge \operatorname{Ric} \leq C_{2} C_{3} R_{i}^{2 \alpha(m-1)-\epsilon} .
$$

Since $2 \alpha(m-1)-\epsilon<0$, if we let $R_{i} \rightarrow \infty$, then

$$
\int_{B_{x_{0}}(1)}(\sqrt{-1} \partial \bar{\partial} u)^{m-1} \wedge \text { Ric }=0
$$

which contradicts (3.3). We conclude that $\operatorname{Ric}\left(x_{0}\right)=0$ and hence Ric $\equiv 0$ on $M \backslash \bigcup_{j=1}^{k} V_{j}$. Therefore, we must have Ric $\equiv 0$.

Suppose $\eta \geq 2$. Then one can choose a $u$ that satisfies (3.1) and (3.2) with $\alpha=0$. Hence, (3.4) becomes

$$
\int_{B_{x_{0}}(1)}(\sqrt{-1} \partial \bar{\partial} u)^{m-1} \wedge \text { Ric } \leq C_{2} R^{-2 m+3} \int_{\partial B_{o}(R)} \mathcal{R} .
$$

Integrating from $R / 2$ to $R$ with $R / 2>r\left(x_{0}\right)+1$, we have

$$
\frac{1}{R} \int_{B_{x_{0}}(1)}(\sqrt{-1} \partial \bar{\partial} u)^{m-1} \wedge \text { Ric } \leq C_{4} \frac{R}{V_{o}(R)} \int_{B_{o}(R)} \mathcal{R}
$$

for some positive constant $C_{4}$ independent of $R$. Here we have used the fact that $V_{o}(R) \sim R^{2 m}$. Integrating again from $2\left(r\left(x_{0}\right)+1\right)$ to $R$, we have

$$
\begin{aligned}
\log \frac{R}{2\left(r\left(x_{0}\right)+1\right)} \int_{B_{x_{0}}(1)}(\sqrt{-1} \partial \bar{\partial} u)^{m-1} \wedge \operatorname{Ric} & \leq C_{4} \int_{0}^{R} \frac{t}{V_{o}(t)} \int_{B_{o}(t)} \mathcal{R} \\
& =o(\log R) .
\end{aligned}
$$

Letting $R \rightarrow \infty$, we have (3.5) again. We conclude that $M$ is Ricci flat as before.

Remark 3.1. (i) The condition ( $\left.\mathrm{b}^{\prime}\right)$ is satisfied if

$$
\lim _{r \rightarrow \infty} \frac{r^{2}}{V_{o}(r)} \int_{B_{o}(r)}|\mathcal{R}|=0 .
$$

(ii) By Remark 2.1, the assumption that $M$ is ALE can be replaced by the assumption that $M$ has finitely many ends such that each end is ALE. Of course, in case $M$ has nonnegative Ricci curvature, then $M$ must have only one end by C-G. 
Applying Theorems 1.1 and 1.5 of [B-K-N] we have the following corollary.

Corollary 3.1. Let $M$ be a complete Kähler manifold of complex dimension $m \geq 2$ such that

(a) $|R m|(x) \leq C r^{-2-\epsilon}(x)$ for some constant $C>0$, where $R m$ is the Riemannian curvature tensor;

(b) $V_{o}(r) \geq C r^{2 m}$ for some positive constant $C>0$; and

(c) either $\operatorname{Ric}(M) \geq 0$ everywhere or $\operatorname{Ric}(M) \leq 0$ everywhere.

Then $M$ is Ricci-flat and $M$ is ALE of order $2 m$.

Proof. By the result of $\mathrm{Ab}$, (a) implies that $M$ has only finitely many ends. By Theorem 1.1 of [B-K-N], each end of $M$ is ALE. Hence, $M$ is Ricci flat by Theorem 3.1 and Remark 3.1. By (a) and the fact that $M$ is ALE,

$$
\int_{M}|R m|^{m}<\infty
$$

By Theorem 1.5 of $[\mathrm{B}-\mathrm{K}-\mathrm{N}]$, we conclude that $M$ is ALE of order $2 m$.

If we replace the assumption (c) on the Ricci curvature by holomorphic bisectional curvature, then we have:

Corollary 3.2. Let $M$ be a complete noncompact Kähler manifold of complex dimension $m \geq 2$. Assume that

(a) $|\mathcal{R}|(x) \leq C r^{-2-\epsilon}(x)$ for some constant $C>0$ where $\mathcal{R}$ is the scalar curvature;

(b) $V_{o}(r) \geq C r^{2 m}$; and

(c) either $M$ has nonnegative holomorphic bisectional curvature or $M$ has nonpositive holomorphic bisectional curvature.

Then $M$ is isometrically biholomorphic to $\mathbb{C}^{m}$.

Proof. (a) and (c) imply that $|R m|(x) \leq C r^{-2-\epsilon}(x)$ for some constant $C$. The result follows from Corollary 3.1.

We should mention that when $M$ has nonnegative holomorphic bisectional curvature, our result is only a special case of Chen and Zhu [C-Z]; see also [S].

It turns out that the method used in the proof of Theorem 3.1 can give a simple but very different proof of a result of Moore [Me, Theorem 2], which is also related to a result of Kasue $\mathrm{Ka}$ :

Theorem 3.3 (Moore). Let $M^{m}$ be a complete complex immersed submanifold of $\mathbb{C}^{N}$ with complex dimension $m \geq 2$. Suppose that $\int_{M}|A|^{2 m}<\infty$, where $|A|$ is the norm of the second fundamental form of $A$ of $M$. Then $M$ is a complex linear subspace.

Proof. Let $z=\left(z^{1}, \ldots, z^{N}\right)$ be the standard complex coordinates in $\mathbb{C}^{N}$ with $|z|=$ $\left[\sum_{i}^{N}\left|z_{i}\right|^{2}\right]^{\frac{1}{2}}$ and let $r(z), z \in M$ be the geodesic distance of $z$ to a fixed point $o \in M$. Since $M$ is minimal, by Proposition 2.1 in $\mathrm{An}$,

$$
|A|(z) \leq|z|^{-1} \tilde{\sigma}(|z|),
$$

where $\tilde{\sigma}(t) \rightarrow 0$ as $t \rightarrow \infty$, and by the proof of [An, Lemma 2.4] there exists a constant $C>0$ such that

$$
C^{-1}|z| \leq r(z) \leq C|z|
$$


for $z \in M$. Combining this with (3.6), we have for $z \in M$,

$$
|A|(z) \leq r^{-1}(z) \sigma(r(z)),
$$

where $\sigma(t) \rightarrow 0$ as $t \rightarrow \infty$. Let $h$ be a smooth function in the ambient space $\mathbb{C}^{N}$. Then we know

$$
|\nabla h| \leq|\bar{\nabla} h|
$$

on $M$. Here $\nabla$ and $\bar{\nabla}$ are the covariant derivatives of $M$ and $\mathbb{C}^{N}$ respectively. Moreover, let $X$ and $Y$ be vector fields on $M$. Then on $M$,

$$
\begin{aligned}
\operatorname{Hess}_{M} h(X, Y) & =X Y h-\left(\nabla_{X} Y\right) h \\
& =X Y h-\bar{\nabla}_{X}^{Y} h+A(X, Y) h \\
& =\operatorname{Hess}_{\mathbb{C}^{N}} h(X, Y)+A(X, Y) h,
\end{aligned}
$$

where $\operatorname{Hess}_{M}$ and $\operatorname{Hess}_{\mathbb{C}^{N}}$ are the Hessians of $h$ with respect to $M$ and $\mathbb{C}^{N}$ respectively. Using (3.8), we have for $z \in M$,

$$
\left|\operatorname{Hess}_{M} h\right|(z) \leq C_{1}\left(\left|\operatorname{Hess}_{\mathbb{C}^{N}} h\right|(z)+r^{-1}(z)|\bar{\nabla} h|(z)\right) .
$$

Let $h=\log \left(1+|z|^{2}\right)=\log \left(1+\sum_{j=1}^{N}\left|z^{j}\right|^{2}\right)$. Then $h$ is strictly plurisubharmonic on $\mathbb{C}^{N}$ and hence on $M$, because $M$ is a complex submanifold. By (3.9), (3.10) and (3.7), there exist constants $C_{2}, C_{3}>0$ such that for any $z \in M$,

$$
|\nabla h|(z) \leq|\bar{\nabla} h|(z) \leq C_{2} r^{-1}(z)
$$

and

$$
\left|\operatorname{Hess}_{M} h\right|(z) \leq C_{3} r^{-2}(z) .
$$

By (3.8), we conclude that the curvature tensor of $M$ satisfies

$$
\left|R m_{M}(z)\right| \leq C_{4} r^{-2}(z) \sigma(r(z)),
$$

for some constant $C_{4}>0$ and by Theorem 3.1 in [An],

$$
V_{o}(r) \leq C r^{2 m},
$$

for some constant $C$. Hence, there exist $R_{i} \rightarrow \infty$ and $C_{5}>0$, such that the area of $\partial B_{o}\left(R_{i}\right)$ is no greater than $C_{5} R_{i}^{2 m-1}$. Then we can proceed as in the proof of Theorem 3.1, since the holomorphic bisectional curvature of $M$ is nonpositive. Namely, let $z_{0} \in M$ and suppose the $\operatorname{Ric}_{M}\left(z_{0}\right) \neq 0$. Using (3.11), (3.12) and (3.13), as in the proof of (3.4), there is a constant $C_{6}$ such that if $R_{i}$ is large enough,

$$
\begin{aligned}
0 & <C_{6} R_{i}^{-1} \cdot R_{i}^{-2(m-2)} \int_{\partial B_{o}\left(R_{i}\right)}|\mathcal{R}| \\
& \leq C_{7} \sigma\left(R_{i}\right)
\end{aligned}
$$

where $\mathcal{R}$ is the scalar curvature of $M$. Letting $i \rightarrow \infty$, we have $\sigma\left(R_{i}\right) \rightarrow 0$. We have a contradiction. Hence, $M$ must be flat and is an affine complex linear subspace. 


\section{§4. Ricci flatness of ALE metrics And the group $G$}

In this section, we will discuss Ricci flatness of an ALE Kähler manifold from another perspective. Let $M^{m}$ be a complete noncompact Kähler manifold with Kähler metric $g$ and with nonnegative Ricci curvature. In this section, we always assume that $M^{m}$ is ALE of order $\lambda(t)$ which satisfies

$$
\begin{cases}\lim _{t \rightarrow \infty} \lambda(t) & =0, \text { and } \\ \int_{1}^{\infty} t^{-1} \lambda(t) d t & <\infty\end{cases}
$$

Let $G$ be the group in the definition of an ALE manifold in $\S 1$. We will give a characterization of Ricci flatness in terms of $G$. In particular, we will replace condition (b) or $\left(\mathrm{b}^{\prime}\right)$ in Theorem 3.1 by a condition on $G$. Moreover, we will only assume $\lambda(t)$ to satisfy (4.1), instead of assuming $\lambda(t)=t^{-\eta}$ for some $\eta>0$ as in Theorem 3.1. For example, $\lambda(t)$ may be of the form $t^{-1}(\log t)^{-1-\epsilon}$ for some $\epsilon>0$. Using the notation in $\S 1$, recall that we have diffeomorphisms

$$
\psi:\left(\mathbb{R}^{2 m} \backslash B_{0}\left(R_{0}\right)\right) / G \rightarrow M \backslash K
$$

and

$$
\phi=\psi \circ \pi: \mathbb{R}^{n} \backslash B_{0}(R) \rightarrow M \backslash K .
$$

Here $R_{0}>0$ is a fixed number and $K$ is a compact set. Let $h=\phi^{*} g$ be the pullback metric. Then the definition of ALE provides

$$
\begin{cases}\left|h_{i j}(x)-\delta_{i j}\right| & \leq C \lambda(|x|), \\ \left|\frac{\partial h_{i j}(x)}{\partial x^{k}}\right| & \leq C|x|^{-1} \lambda(|x|), \\ \left|\Gamma_{i j}^{k}(x)\right| & \leq C|x|^{-1} \lambda(|x|)\end{cases}
$$

for some constant $C$.

First, we construct the complex structure on the tangent cone $\left(\mathbb{R}^{2 m} \backslash B_{0}\left(R_{0}\right)\right) / G$. Let $J_{M}$ be the complex structure on $M$ and let $J$ be the pullback of $J_{M}$ on $\mathbb{R}^{2 m} \backslash$ $B_{0}\left(R_{0}\right)$. Namely, $J=\phi_{*}^{-1} \circ J_{M} \circ \phi_{*}$, where the differential $\phi_{*}$ of $\phi$ is an isomorphism at each point. In the standard coordinates $\left(x^{1}, \ldots, x^{2 m}\right)$ on $\mathbb{R}^{2 m} \backslash B_{0}(R)$, we can write

$$
J(x)=\sum_{i, j} J_{j}^{i}(x) \frac{\partial}{\partial x^{i}} \otimes d x^{j}
$$

and hence $J(x)\left(\frac{\partial}{\partial x^{i}}\right)=J_{i}^{j}(x) \frac{\partial}{\partial x^{j}}$, with $\left(J_{i}^{j}\right)^{2}=-I$ where $I$ is the identity matrix.

Lemma 4.1. With the above assumptions and notation, the following are true:

(a) $\lim _{x \rightarrow \infty} J_{i}^{j}(x)=\tilde{J}_{i}^{j}$ for all $i, j$ such that

$$
\left|J_{i}^{j}(x)-\tilde{J}_{i}^{j}\right| \leq C\left[\lambda(|x|)+\int_{|x|}^{\infty} t^{-1} \lambda(t) d t\right],
$$

for some constant $C$ for all $x$, where $\tilde{J}_{i}^{j}$ are constants.

(b)

$$
\left|\frac{\partial J_{j}^{k}}{\partial x^{i}}\right|(x) \leq C|x|^{-1} \lambda(|x|),
$$

for some constant $C$ for all $x$ and $1 \leq i, j, k \leq 2 m$.

(c) If $\tilde{J}$ is the matrix $\left(\tilde{J}_{i}^{j}\right)$, then $\tilde{J}^{2}=-\bar{I}$.

(d) $\tilde{J} \in O(2 m)$ with respect to the standard metric on $\mathbb{R}^{2 m}$. 
(e) For any $\gamma \in G$, we have $\gamma \circ \tilde{J}=\tilde{J} \circ \gamma$.

Proof. Since $h$ is the pullback metric of $g$ which is Kähler, if $\nabla$ is the covariant differentiation with respect to $h$, then

$$
\begin{aligned}
0 & =\nabla_{\frac{\partial}{\partial x^{i}}} J_{k}^{j} \\
& =\frac{\partial J_{k}^{j}}{\partial x^{i}}+\Gamma_{i p}^{j} J_{k}^{p}-\Gamma_{i k}^{p} J_{p}^{j}
\end{aligned}
$$

where $\Gamma_{i j}^{k}$ are the Christoffel symbols of $h$ with respect to the coordinates $x^{i}$. By (4.2), we have

$$
\left|\frac{\partial J_{k}^{j}}{\partial x^{i}}\right|(x) \leq C_{1}|x|^{-1} \lambda(|x|)|| J||(x),
$$

for some constant $C_{1}$, where $\|J\|^{2}=\sum_{i j}\left(J_{i}^{j}\right)^{2}$. Since $t^{-1} \lambda(t)$ is integrable near infinity, one can conclude that $\|J\|$ is bounded. From this and (4.3), (b) follows.

To prove (a), let $x$ and $x^{\prime}$ be two points, such that $|x| \geq\left|x^{\prime}\right|$; by (b) there exists a constant $C_{2}$ independent of $x$ and $x^{\prime}$ such that

$$
\left|J_{i}^{j}(x)-J_{i}^{j}\left(x^{\prime}\right)\right| \leq C_{2}\left(\lambda\left(\left|x^{\prime}\right|\right)+\int_{\left|x^{\prime}\right|}^{|x|} t^{-1} \lambda(t) d t\right) .
$$

From this and (4.1), (a) follows. (c) follows from the fact that $J^{2}=-I$. Note that

$$
\left\langle J\left(\frac{\partial}{\partial x^{i}}\right), J\left(\frac{\partial}{\partial x^{j}}\right)\right\rangle=h_{i j} .
$$

The right side of the equality tends to $\delta_{i j}$ as $x \rightarrow \infty$. From this (d) follows. Since $J$ is the pullback of $J_{M}, \gamma \circ J \circ \gamma^{-1}=J$ for $\gamma \in G$. Here $\gamma$ can be considered as a constant matrix. Hence (e) is true.

By (c) and (d) we can conclude that $\tilde{J}=S \circ J_{0} \circ S^{-1}$ for some $S \in O(2 m)$, where $J_{0}$ is the standard complex structure on $\mathbb{R}^{2 m}$, namely

$$
J_{0}\left(\frac{\partial}{\partial x^{i}}\right)=\frac{\partial}{\partial x^{m+i}}
$$

and

$$
J_{0}\left(\frac{\partial}{\partial x^{m+i}}\right)=-\frac{\partial}{\partial x^{i}}
$$

for $1 \leq i \leq m$. Hence, by conjugating with $S$ we may assume that $\tilde{J}$ is the standard complex structure. For simplicity, we call $\tilde{J}$ the complex structure of the tangent cone of $M$. With respect to this complex structure, $G \subset U(m)$. The following proposition may be well known.

Proposition 4.1. With the above notation, suppose $M^{m}$ is a complete noncompact ALE Kähler manifold with group $G$ and of order $\lambda(t)$ satisfying (4.1). Suppose $M$ is simply connected and Ricci flat. Then $G$ is a subgroup of $S U(m)$ with respect to the complex structure on the tangent cone of $M$.

Proof. Since $M$ is simply connected and Ricci flat, there is a nontrivial parallel $(m, 0)$ form $\Theta$ on $M$ by [Be, Proposition 10.29]. The pullback of $\Theta$ on $\mathbb{R}^{2 m} \backslash B_{0}\left(R_{0}\right)$ 
is denoted by the same notation. Then $\Theta$ is also a nontrivial parallel $(m, 0)$ with respect to the complex structure $J$. For $\tau_{i} \rightarrow \infty$, let

$$
\Theta_{i}(x)=\tau_{i}^{-m} \Theta\left(\tau_{i} x\right) .
$$

We claim that by passing to a subsequence, $\Theta_{i} \rightarrow \Theta_{\infty}$ uniformly on compact subsets of $\mathbb{R}^{2 m} \backslash B_{0}\left(R_{0}\right)$. Moreover, $\Theta_{\infty}$ is invariant under $G$, is parallel with respect to the Euclidean metric and is nontrivial.

Indeed, if we write

$$
\Theta=\sum_{|I|=m} f_{I} d x^{I}
$$

where $I$ is a multi-index $d x^{I}=d x^{i_{1}} \otimes \cdots \otimes d x^{i_{m}}$ which form a basis for the tensor fields, then since $\Theta$ is parallel, by (4.1) and (4.2), we have

$$
C_{1}^{-1}\|\Theta\|(x) \leq \sum_{I}\left|f_{I}\right|^{2}(x) \leq C_{1}\|\Theta\|(x)
$$

for some positive constant $C_{1}>0$ independent of $x$. Here $\|\Theta\|$ is the norm of $\Theta$ with respect to $h$. In particular, $\left|f_{I}\right|$ are uniformly bounded because $\|\Theta\|$ is a constant. Since $\Theta$ is parallel with respect to $h$, by (4.2) and the definition of covariant derivative, we have

$$
\left|\frac{\partial}{\partial x^{i}} f_{I}(x)\right| \leq C_{2}|x|^{-1} \lambda(|x|) .
$$

Now

$$
\Theta_{i}(x)=\sum_{I} f_{I}\left(\tau_{i} x\right) d x^{I}=\sum_{I} f_{i, I}(x) d x^{I},
$$

where $f_{i, I}(x)=f_{I}\left(\tau_{i} x\right)$. By $(4.5)$

$$
\left|\frac{\partial}{\partial x^{i}} f_{i, I}(x)\right| \leq C_{2} \tau_{i}\left|\tau_{i} x\right|^{-1} \lambda\left(\tau_{i}|x|\right)=C_{2}|x|^{-1} \lambda\left(\tau_{i}|x|\right) .
$$

In particular, $f_{i, I}$ are equicontinuous on compact sets. Passing to a subsequence, if necessary, we conclude that the $\Theta_{i}$ converge uniformly on compact sets in $\mathbb{R}^{2 m} \backslash$ $B_{0}\left(R_{0}\right)$. By (4.6), passing to a subsequence, we have $f_{i, I} \rightarrow a_{I}$, which is a constant. Note that $\Theta$ and hence $\Theta_{i}$ is invariant under $G$. Hence $\Theta_{\infty}=\sum_{|I|=m} a_{I} d x^{I}$ is also invariant under $G$. By (4.4), we know that $\Theta_{\infty}$ is nontrivial. It is obviously parallel with respect to the Euclidean metric because $a_{I}$ are constants. We may assume that the complex structure of the tangent cone of $M$ is the standard one $J_{0}$; that is,

Let us write

$$
J_{0}\left(d x^{j}\right)=-d x^{m+j} ; \quad J_{0}\left(d x^{m+j}\right)=d x^{j} .
$$

$$
J\left(d x^{i}\right)=J_{i}^{j} d x^{j}
$$

and

$$
J_{0}\left(d x^{i}\right)=\tilde{J}_{i}^{j} d x^{j} .
$$

Let $e^{j}=d x^{j}-\sqrt{-1} J\left(d x^{j}\right), e_{0}^{j}=d x^{j}-\sqrt{-1} J_{0}\left(d x^{j}\right), 1 \leq j \leq m$. It is easy to see that $e^{1}, \ldots, e^{m}$ form a basis for the $(1,0)$ forms with respect to $J$. Let $\tilde{e}_{i}^{j}(x)=d x^{j}-\sqrt{-1} J\left(\tau_{i} x\right) d x^{j}$. Then by Lemma 4.1(a),

$$
\left|e_{0}^{j}-\tilde{e}_{i}^{j}(x)\right| \leq C_{3} \tilde{\lambda}\left(\left|\tau_{i} x\right|\right),
$$

where $\tilde{\lambda}(t) \rightarrow 0$ as $t \rightarrow \infty$. Since $\Theta$ is a holomorphic $(m, 0)$ form with respect to $J$,

$$
\Theta=\phi(x) e^{1} \wedge \cdots \wedge e^{m}
$$


and

$$
\Theta_{i}(x)=\phi\left(\tau_{i} x\right) \tilde{e}_{i}^{1} \wedge \cdots \wedge \tilde{e}_{i}^{m} .
$$

Note that by (4.2) and the fact that $\|\Theta\|$ is a constant, $\phi$ is bounded. By (4.6) and (4.1), we have

$$
\Theta_{i}(x)=\phi\left(\tau_{i} x\right) e_{0}^{1} \wedge \cdots \wedge e_{0}^{m}+\eta_{i}
$$

where $\left|\eta_{i}(x)\right| \leq C_{3} \tilde{\lambda}\left(\left|\tau_{i} x\right|\right)$ for some constant $C_{3}$. Hence, $\lim _{i \rightarrow \infty} \eta_{i}(x) \rightarrow 0$ and $\Theta_{\infty}=a e_{0}^{1} \wedge \cdots \wedge e_{0}^{m}$ for some constant $a$ which is nonzero because of (4.4). Since $\Theta_{\infty}$ is invariant under $G$, we must have $\operatorname{det} \gamma=1$ for all $\gamma \in G$. Since $G \subset U(m)$ with respect to $J_{0}$, we conclude that $G \subset S U(m)$.

In case $M$ is an ALE Kähler manifold with nonnegative Ricci curvature, one may replace the condition on curvature decay in Theorem 3.1 by a reasonable condition on $G$. More precisely, we will prove that $G$ being in $S U(m)$ is also sufficient for the Ricci flatness of $M$. We will need various forms of Bochner-Weitzenböck formulae. Let $\Delta_{d}=d \delta+\delta d$, where $\delta$ is the adjoint of $d$ with respect to the Riemannian metric on $M$. Let $\Delta_{\bar{\partial}}=\bar{\partial} \bar{\partial}^{*}+\bar{\partial}^{*} \bar{\partial}$, where $\bar{\partial}^{*}$ is the adjoint of $\bar{\partial}$ with respect to the Kähler metric on $M$. Then $\frac{1}{2} \Delta_{d}=\Delta_{\bar{\partial}}$. In this notation, for a function $f$ in $\mathbb{R}^{n}$, $\Delta_{d} f=-\sum_{i}^{n} \frac{\partial^{2}}{\partial x_{i}^{2}} f$. The following is well known.

Lemma 4.2. Let $M^{m}$ be a Kähler manifold.

(a) If $\phi$ is an $(m, 0)$ form, then

$-\Delta_{d}\|\phi\|^{2}(x)=2\|\nabla \phi\|^{2}(x)+\left\langle-\Delta_{d} \phi, \phi\right\rangle(x)+\left\langle\phi,-\Delta_{d} \phi\right\rangle(x)+\mathcal{R}(x)\|\phi\|^{2}(x)$, where $\mathcal{R}(x)$ is the scalar curvature of $M$ at $x$.

(b) If $\phi$ is an $(m, 1)$ form, then

$$
-\Delta_{d}\|\phi\|^{2}(x) \geq 2\|\nabla \phi\|^{2}(x)+\left\langle-\Delta_{d} \phi, \phi\right\rangle(x)+\left\langle\phi,-\Delta_{d} \phi\right\rangle(x)+\mathcal{R}_{m-1}(x)\|\phi\|^{2}(x),
$$

where $\mathcal{R}_{m-1}(x)=\lambda_{1}(x)+\cdots+\lambda_{m-1}(x)$ with $\lambda_{1} \leq \lambda_{2} \leq \cdots \leq \lambda_{m}$ are the eigenvalues of the Ricci form $R_{i \bar{j}} d z_{i} \wedge d \bar{z}_{j}$ at the point $x$.

Here the inner products are taken with respect to the Kähler metric.

Proof. Choose normal coordinates around $x \in M$. By Theorem 6.1 and Theorem 6.2 of $[\mathrm{M}-\mathrm{K}]$, for an $(m, 0)$ form $\phi$,

$$
\frac{1}{2} \Delta_{d} \phi=\Delta_{\bar{\partial}} \phi=-\nabla_{i} \nabla_{\bar{i}} \phi
$$

and

$$
\frac{1}{2} \Delta_{d} \phi=\Delta_{\bar{\partial}} \phi=-\nabla_{\bar{i}} \nabla_{i} \phi+\mathcal{R}(x) \phi .
$$

Combining the above two equalities with

$$
\begin{aligned}
-\Delta_{d}\|\phi\|^{2} & =2\langle\phi, \phi\rangle_{\bar{i} \bar{i}} \\
& =2\left\|\nabla_{i} \phi\right\|^{2}+2\left\|\nabla_{\bar{i}} \phi\right\|^{2}+\left\langle 2 \nabla_{i} \nabla_{\bar{i}} \phi, \phi\right\rangle+\left\langle\phi, 2 \nabla_{\bar{i}} \nabla_{i} \phi\right\rangle
\end{aligned}
$$

we have (a). If $\phi$ is an $(m, 1)$ form, then we write $\phi=\phi_{\bar{k}} d z_{1} \wedge \cdots \wedge d z_{m} \wedge d \bar{z}_{k}$. Theorem 6.1 and Theorem 6.2 of [M-K] again imply that

$$
\left(\frac{1}{2} \Delta_{d} \phi\right)_{\bar{k}}=\left(\Delta_{\bar{\partial}} \phi\right)_{\bar{k}}=-\left(\nabla_{i} \nabla_{\bar{i}} \phi\right)_{\bar{k}}
$$

and

$$
\left(\frac{1}{2} \Delta_{d} \phi\right)_{\bar{k}}=\left(\Delta_{\bar{\partial}} \phi\right)_{\bar{k}}=-\left(\nabla_{\bar{i}} \nabla_{i} \phi\right)+\mathcal{R} \phi_{\bar{k}}-\sum R_{k \bar{l}} \phi_{\bar{l}} .
$$

Then a similar calculation proves (b). 
Theorem 4.1. Let $M^{m}(m \geq 2)$ be a complete noncompact ALE Kähler manifold with group $G$ and of order $\lambda$ such that $\lambda$ satisfies (4.1). Suppose that

(a) $M$ has nonnegative Ricci curvature; and

(b) $G$ is a subgroup of $S U(m)$ with respect to the complex structure on the tangent cone.

Then $M$ is Ricci flat.

Proof. By [Be, Proposition 10.29], it is sufficient to construct a nontrivial parallel holomorphic $(m, 0)$ form on $M$. To this end, we first construct an "almost" parallel $(m, 0)$ form on $M$.

Using the notation as in the beginning of this section, let $\left(x^{1}, \ldots, x^{2 m}\right)$ be the standard coordinates in $\mathbb{R}^{2 m}$. We may assume that the complex structure $J_{0}$ on the tangent cone is the standard one such that

$$
J_{0}\left(d x^{j}\right)=-d x^{m+j}, \quad J_{0}\left(d x^{m+j}\right)=d x^{j},
$$

for $1 \leq j \leq m$. Let $\omega_{0}=e_{0}^{1} \wedge \cdots \wedge e_{0}^{m}$ where $e_{0}^{j}=d x^{j}-\sqrt{-1} J_{0}\left(d x^{j}\right)$. Let $\tilde{\omega}=e^{1} \wedge \cdots \wedge e^{m}$, where $e^{j}=d x^{j}-\sqrt{-1} J\left(d x^{j}\right), 1 \leq j \leq m$. Then $\tilde{\omega}$ is an $(m, 0)$ form with respect to $J$. By Lemma 4.1, we conclude that $\tilde{\omega}$ is never zero near infinity. Let

$$
\omega=\|\tilde{\omega}\|^{-2}\left\langle\omega_{0}, \tilde{\omega}\right\rangle \tilde{\omega}
$$

where the inner product and the norm are taken with respect to the metric $h$. Since $G \subset S U(m)$ with respect to $J_{0}, \omega_{0}$ descends to an $m$ form on $M \backslash K$. Since $\omega$ is just the $(m, 0)$ part of $\omega_{0}$ with respect to $J$ which is the pullback of $J_{M}, \omega$ also descends to $M$ near infinity. We will also denote this form by $\omega$. Let $\tilde{x}, \tilde{y}, \ldots$, etc., denote points on $M$. Let us first assume that $\lambda$ is nonincreasing. By Lemma 4.1, (4.2) and Lemma 2.1, we have

$$
\lim _{\tilde{x} \rightarrow \infty}\|\omega\|=1
$$

and

$$
\|\nabla \omega\|(\tilde{x})+\|d \omega\|(\tilde{x})+\|\delta \omega\|(\tilde{x}) \leq C_{1} r^{-1}(\tilde{x}) \tilde{\lambda}(r(\tilde{x}))
$$

for some constant $C_{1}$ independent of $\tilde{x}$, where $\tilde{\lambda}$ is a nonnegative function on $[0, \infty)$ satisfying (4.1). Extend $\omega$ to be a smooth $(m, 0)$ form on $M$ so that it equals to $\omega$ outside a compact set. We still denote this form by $\omega$.

For any $R>0$, by $\left[\mathrm{My}\right.$ p. 316, Theorem 7.8.4], there is a unique $m$ form $\Theta_{R}$ on $B_{o}(R)$, where $o$ is a fixed point in $M$, such that

$$
\left\{\begin{array}{lll}
\Delta_{d} \Theta_{R} & =0 & \text { on } B_{o}(R), \\
\Theta_{R} & =\omega & \text { on } \partial B_{o}(R) .
\end{array}\right.
$$

It is easy to see that $\Theta_{R}$ is an $(m, 0)$ form because $\Delta_{d}$ is a real operator. Since $\left\|\Theta_{R}\right\|^{2}$ is subharmonic by the Bochner-Weitzenböck formula (Lemma 4.2(a)), we have

$$
\sup _{B_{o}(R)}\left\|\Theta_{R}\right\|^{2} \leq \sup _{\partial B_{o}(R)}\left\|\Theta_{R}\right\|^{2}=\sup _{\partial B_{o}(R)}\|\omega\|^{2} \leq C_{2}
$$

for some constant $C_{1}$ independent of $R$. By the interior Schauder estimates of elliptic systems $\mathrm{My}$. Theorem 5.5.3], after passing to a subsequence if necessary, $\Theta_{R}$ converges uniformly on compact sets in $M$ to an $(m, 0)$ form $\Theta$. We want to prove that $\Theta$ is nontrivial, holomorphic, and parallel. 
To prove $\Theta$ is nontrivial, let $\omega_{R}=\Theta_{R}-\omega$. Then $\Delta_{d} \omega_{R}=-\Delta_{d} \omega$ in $B_{o}(R)$ and $\omega_{R}=0$ on $\partial B_{o}(R)$. On the other hand, by the Bochner-Weitzenböck formula in Lemma 4.2(a), we have

$$
\begin{aligned}
-\Delta_{d}\left\|\omega_{R}\right\|^{2} & =2\left\|\nabla \omega_{R}\right\|^{2}+\left\langle-\Delta_{d} \omega, \omega_{R}\right\rangle+\left\langle\omega_{R},-\Delta_{d} \omega\right\rangle+2 \mathcal{R}(x)\left\|\omega_{R}\right\|^{2} \\
& \geq 2\left\|\nabla \omega_{R}\right\|^{2}+\left\langle-\Delta_{d} \omega, \omega_{R}\right\rangle+\left\langle\omega_{R},-\Delta_{d} \omega\right\rangle
\end{aligned}
$$

where we have used the fact that $\mathcal{R}(x)$, the scalar curvature of $M$, is nonnegative and that $\Delta_{\bar{\partial}}=\frac{1}{2} \Delta_{d}$. Note that here $\Delta_{d}=d \delta+\delta d$ on functions differs from the usual Beltrami-Laplace operator by a minus sign. Define

$$
\begin{aligned}
u(\tilde{x})= & -\int_{B_{o}(R)} G_{R}(\tilde{x}, \tilde{y})\left(2\left\|\nabla \omega_{R}\right\|^{2}(\tilde{y})+\left\langle-\Delta_{d} \omega(\tilde{y}), \omega_{R}(\tilde{y})\right\rangle\right. \\
& \left.+\left\langle\omega_{R}(\tilde{y}),-\Delta_{d} \omega(\tilde{y})\right\rangle\right) d \tilde{y}
\end{aligned}
$$

where $G_{R}$ is the positive Green's function on $B_{o}(R)$ with zero boundary value. Since $-\Delta_{d}\left\|\omega_{R}\right\|^{2} \geq-\Delta_{d} u$ and $\left\|\omega_{R}\right\|^{2}=u=0$ on $\partial B_{o}(R), u \geq\left\|\omega_{R}\right\|$ on $B_{o}(R)$. On the other hand, for $\tilde{x} \in B_{o}\left(\frac{1}{8} R\right)$, integrating by parts in (4.10), noting that the singularity of $G_{R}$ at $\tilde{x}$ does not cause any trouble, we have

$$
\begin{aligned}
u(\tilde{x}) \leq & -2 \int_{B_{o}(R)} G_{R}(\tilde{x}, \tilde{y})\left\|\nabla \omega_{R}\right\|^{2} d \tilde{y} \\
& +2 \int_{B_{o}(R)}\left(\left\|d \omega_{R}\right\|(\tilde{y})+\left\|\delta \omega_{R}\right\|(\tilde{y})\right)(\|d \omega\|(\tilde{y})+\|\delta \omega\|(\tilde{y})) G_{R}(\tilde{x}, \tilde{y}) d \tilde{y} \\
& +C_{3} \int_{B_{o}(R)}\left|\nabla_{\tilde{y}} G_{R}(\tilde{x}, \tilde{y})\right|\left\|\omega_{R}\right\|(\tilde{y})(\|d \omega\|(\tilde{y})+\|\delta \omega\|(\tilde{y})) d \tilde{y} \\
\leq & C_{4}\left(\int_{B_{o}(R)}\left(\|d \omega\|^{2}(\tilde{y})+\|\delta \omega\|^{2}(\tilde{y})\right) G_{R}(\tilde{x}, \tilde{y}) d \tilde{y}\right. \\
& +\int_{B_{o}\left(\frac{1}{2} R\right)}(\|d \omega\|(\tilde{y})+\|\delta \omega\|(\tilde{y})) \frac{G_{R}(\tilde{x}, \tilde{y})}{r(\tilde{x}, \tilde{y})} d \tilde{y} \\
& \left.+\int_{B_{o}(R) \backslash B_{o}\left(\frac{1}{2} R\right)}\left|\nabla_{\tilde{y}} G_{R}(\tilde{x}, \tilde{y})\right|(\|d \omega\|(\tilde{y})+\|\delta \omega\|(\tilde{y})) d \tilde{y}\right) \\
\leq & C_{5}\left(\int_{B_{o}(R)}\left(\|d \omega\|^{2}(\tilde{y})+\|\delta \omega\|^{2}(\tilde{y})\right) r^{2-2 m}(\tilde{x}, \tilde{y}) d \tilde{y}\right. \\
& +\int_{B_{o}\left(\frac{1}{2} R\right)}(\|d \omega\|(\tilde{y})+\|\delta \omega\|(\tilde{y})) r^{1-2 m}(\tilde{x}, \tilde{y}) d \tilde{y} \\
& \left.+\int_{B_{o}(R) \backslash B_{o}\left(\frac{1}{2} R\right)}\left|\nabla_{\tilde{y}} G_{R}(\tilde{x}, \tilde{y})\right|(\|d \omega\|(\tilde{y})+\|\delta \omega\|(\tilde{y})) d \tilde{y}\right) \\
\leq & C_{6}\left(\int_{B_{o}(R)}\left[r^{-2}(\tilde{y}) \tilde{\lambda}^{2}(r(\tilde{y})) r^{2-2 m}(\tilde{x}, \tilde{y})+r^{-1}(\tilde{y}) \tilde{\lambda}(r(\tilde{y})) r^{1-2 m}(\tilde{x}, \tilde{y})\right] d \tilde{y}\right. \\
& \left.+R^{-1} \sup _{t \geq \frac{1}{2} R} \tilde{\lambda}(t) \int_{B_{o}(R) \backslash B_{o}\left(\frac{1}{2} R\right)}\left|\nabla_{\tilde{y}} G_{R}(\tilde{x}, \tilde{y})\right|\right)
\end{aligned}
$$

for some constants $C_{3}-C_{6}$ independent of $R$. Here we have used (4.8), the fact that $G_{R}(\tilde{x}, \tilde{y}) \leq G(\tilde{x}, \tilde{y}),\left|\nabla_{y} G_{R}(\tilde{x}, \tilde{y})\right| \leq C G_{R}(\tilde{x}, \tilde{y}) r^{-1}(\tilde{x}, \tilde{y})$, Li and Yau's [L-Y] estimate on the Green's function, the fact that $M$ has maximal volume growth, and that $\left\|\omega_{R}\right\|,\|\omega\|$ are bounded (by (4.7) and (4.9)). We have also used the fact that 
$\left\|d \omega_{R}\right\|$ and $\left\|\delta \omega_{R}\right\|$ are bounded by $C\left\|\nabla \omega_{R}\right\|$ for some constant depending only on $m$ (see [DR, p. $107(2)$, p. $108(3)]$ ). Let $r=r(\tilde{x})$, the distance of $\tilde{x}$ from $o$. We want to estimate

$$
\int_{B_{o}(R)} r^{-1}(\tilde{y}) \tilde{\lambda}(r(\tilde{y})) r^{1-2 m}(\tilde{x}, \tilde{y}) d \tilde{y}
$$

for $R \geq 4 r$, where $r=r(\tilde{x})$. Let $1 / 4 \geq \epsilon>0$. Then

$$
\int_{B_{\tilde{x}}\left(\frac{r}{2}\right)} r^{-1}(\tilde{y}) \tilde{\lambda}(r(\tilde{y})) r^{1-2 m}(\tilde{x}, \tilde{y}) d \tilde{y} \leq C_{7} \sup _{t \geq \frac{r}{2}} \tilde{\lambda}(t)
$$

for some constant $C_{1}$ independent of $R, \tilde{x}$ and $\epsilon$.

$$
\begin{aligned}
\int_{B_{o}\left(\frac{r}{2}\right)} & r^{-1}(\tilde{y}) \tilde{\lambda}(r(\tilde{y})) r^{1-2 m}(\tilde{x}, \tilde{y}) d \tilde{y} \\
= & \left(\int_{0}^{\epsilon r}+\int_{\epsilon r}^{\frac{r}{2}}\right)\left(r^{-1}(\tilde{y}) \tilde{\lambda}(r(\tilde{y})) r^{1-2 m}(\tilde{x}, \tilde{y}) d \tilde{y}\right) \\
\quad \leq & C_{8}\left(\epsilon^{2 m-1}+\sup _{t \geq \epsilon r} \tilde{\lambda}(t)\right)
\end{aligned}
$$

for some constant $C_{8}$ independent of $R, \tilde{x}$ and $\epsilon$.

$$
\begin{gathered}
\int_{B_{o}(R) \backslash\left(B_{o}\left(\frac{r}{2}\right) \cup B_{\tilde{x}}\left(\frac{r}{2}\right)\right)} r^{-1}(\tilde{y}) \tilde{\lambda}(r(\tilde{y})) r^{1-2 m}(\tilde{x}, \tilde{y}) d \tilde{y} \\
\leq C_{9} \int_{B_{o}(R) \backslash B_{o}\left(\frac{r}{2}\right)} r^{-2 m}(\tilde{y}) \tilde{\lambda}(r(\tilde{y})) d \tilde{y} \\
\leq C_{9} \int_{\frac{r}{2}}^{\infty} t^{-1} \tilde{\lambda}(t) d t
\end{gathered}
$$

for some constant $C_{9}$ independent of $R, \tilde{x}$ and $\epsilon$. Combining (4.12)-(4.14), if $R \geq 4 r(\tilde{x})$, then

$$
\begin{aligned}
\int_{B_{o}(R)} & r^{-1}(\tilde{y}) \tilde{\lambda}(r(\tilde{y})) r^{1-2 m}(\tilde{x}, \tilde{y}) d \tilde{y} \\
\leq & C_{10}\left[\epsilon^{2 m-1}+\sup _{t \geq \epsilon r} \tilde{\lambda}(t)+\int_{\frac{r}{2}}^{\infty} t^{-1} \tilde{\lambda}(t) d t\right]
\end{aligned}
$$

for some constant $C_{10}$ independent of $R, \tilde{x}$ and $\epsilon$. Similarly, if we choose a possibly larger constant, we also have

$$
\begin{aligned}
\int_{B_{o}(R)} & r^{-2}(\tilde{y}) \tilde{\lambda}^{2}(r(\tilde{y})) r^{2-2 m}(\tilde{x}, \tilde{y}) d \tilde{y} \\
\leq & C_{10}\left[\epsilon^{2 m-2}+\sup _{t \geq \epsilon r} \tilde{\lambda}(t)+\int_{\frac{r}{2}}^{\infty} t^{-1} \tilde{\lambda}(t) d t\right] .
\end{aligned}
$$

Here we have used the fact that $m \geq 2$.

To estimate the last term in (4.11), let $\varphi$ be a nonnegative cutoff function such that $\varphi \equiv 1$ on $B_{o}(R) \backslash B_{o}\left(\frac{1}{2} R\right), \varphi \equiv 0$ on $B_{o}\left(\frac{1}{4} R\right)$ and $|\nabla \varphi| \leq C R^{-1}$ for some constant $C$ independent of $\tilde{x}$ and $R$. Since $G_{R}(\tilde{x}, \cdot)$ is harmonic and smooth on 
$B_{o}(R) \backslash B_{o}\left(\frac{1}{4} R\right)$ with value zero at $\partial B_{o}(R)$, we have

$$
\begin{aligned}
\int_{B_{o}(R) \backslash B_{o}\left(\frac{1}{2} R\right)}\left|\nabla_{\tilde{y}} G_{R}(\tilde{x}, \tilde{y})\right| d \tilde{y} & \leq \int_{B_{o}(R) \backslash B_{o}\left(\frac{1}{4} R\right)} \varphi(\tilde{y})\left|\nabla_{\tilde{y}} G_{R}(\tilde{x}, \tilde{y})\right| d \tilde{y} \\
& \leq C_{11} R^{m}\left(\int_{B_{o}(R) \backslash B_{o}\left(\frac{1}{4} R\right)} \varphi^{2}(\tilde{y})\left|\nabla_{\tilde{y}} G_{R}(\tilde{x}, \tilde{y})\right|^{2} d \tilde{y}\right)^{\frac{1}{2}} \\
& \leq C_{12} R^{m}\left(\int_{B_{o}(R) \backslash B_{o}\left(\frac{1}{4} R\right)}|\nabla \varphi|^{2}(\tilde{y}) G_{R}^{2}(\tilde{x}, \tilde{y}) d \tilde{y}\right)^{\frac{1}{2}} \\
& \leq C_{13} R^{-1+m}\left(\int_{B_{o}(R) \backslash B_{o}\left(\frac{1}{4} R\right)} G^{2}(\tilde{x}, \tilde{y}) d \tilde{y}\right)^{\frac{1}{2}} \\
& \leq C_{14} R
\end{aligned}
$$

for some constants $C_{11}-C_{14}$ which are independent of $\tilde{x}$ and $R$. Here we have used the fact that there is a constant depending only on $m$ such that $G_{R}(\tilde{x}, \tilde{y}) \leq$ $G(\tilde{x}, \tilde{y}) \leq C R^{2-2 m}$ for $\tilde{x} \in B_{o}\left(\frac{1}{8} R\right)$ and $\tilde{y} \notin B_{o}\left(\frac{1}{4} R\right)$. Combining (4.11), (4.15), (4.16) and (4.17), we have

$$
\left\|\omega_{R}\right\|^{2}(\tilde{x}) \leq u(\tilde{x}) \leq C_{15}\left[\epsilon^{2 m-2}+\sup _{t \geq \epsilon r} \tilde{\lambda}(t)+\int_{\frac{r}{2}}^{\infty} t^{-1} \tilde{\lambda}(t) d t+\sup _{t \geq \frac{1}{2} R} \tilde{\lambda}(t)\right]
$$

for some constant $C_{15}$ independent of $R, \tilde{x}$ and $\epsilon$. Let $\epsilon=r^{-\frac{1}{2}}$. If $r \geq 16$, we have

$$
\left\|\omega_{R}\right\|^{2}(\tilde{x}) \leq C_{15}\left[r^{-m+1}+\sup _{t \geq \sqrt{r}} \tilde{\lambda}(t)+\int_{\frac{r}{2}}^{\infty} t^{-1} \tilde{\lambda}(t) d t+\sup _{t \geq \frac{1}{2} R} \tilde{\lambda}(t)\right] .
$$

Since $\tilde{\lambda}(t) \rightarrow 0$ as $t \rightarrow \infty$, if we let $R \rightarrow \infty$, we have

$$
\|\Theta-\omega\|^{2}(\tilde{x}) \leq C_{15}\left[r^{-m+1}+\sup _{t \geq \sqrt{r}} \tilde{\lambda}(t)+\int_{\frac{r}{2}}^{\infty} t^{-1} \tilde{\lambda}(t) d t\right]=\sigma(r)
$$

for all $\tilde{x}$. Since $m \geq 2$ and $\int_{1}^{\infty} t^{-1} \tilde{\lambda}(t) d t<\infty, \sigma(r) \rightarrow 0$ as $r \rightarrow \infty$, combining this with (4.7), we have

$$
\lim _{\tilde{x} \rightarrow \infty}\|\Theta\|(\tilde{x})=1
$$

In particular, $\Theta$ is nontrivial.

Next, we will prove that $\Theta$ is holomorphic. Since $\Delta_{d} \Theta=0$, if $\phi$ is a cutoff function, then

$$
\int_{M}\left\langle\phi^{2} \Theta, \Delta_{d} \Theta\right\rangle=0
$$

Integrating by parts, choosing a suitable cutoff function, by (4.18), we can conclude

$$
\int_{B_{o}(R)}\|d \Theta\|^{2} \leq C_{16} R^{2 m-2}
$$

for some constant $C_{16}$ independent of $R$. Since $\Delta_{\bar{\partial}}=\bar{\partial} \bar{\partial}^{*}+\bar{\partial}^{*} \bar{\partial}=\frac{1}{2} \Delta_{d}$, and $\Theta$ is harmonic, it is easy to see that

$$
\Delta_{\bar{\partial}} \bar{\partial} \Theta=\bar{\partial} \bar{\partial}^{*} \bar{\partial} \Theta=-\bar{\partial}^{*} \bar{\partial} \bar{\partial} \Theta=0 .
$$


Since $\bar{\partial} \Theta$ is a harmonic $(m, 1)$ form and $M$ has nonnegative Ricci curvature, $\|\bar{\partial} \Theta\|^{2}$ is subharmonic by the Bochner-Weitzenböck formula in Lemma 4.2(b). Using the mean value inequality of $\mathrm{Li}$ and Schoen $\mathrm{L}-\mathrm{S}$ ] and (4.19), it is easy to see that $\bar{\partial} \Theta \equiv 0$.

Finally, we want to prove that $\Theta$ is parallel. Since $\Theta$ is a holomorphic $(m, 0)$ form, $M$ has nonnegative Ricci curvature and $\|\Theta\|^{2}$ is plurisubharmonic. Since $M$ is ALE of order $\lambda(t)$, by Proposition 2.1(a), $M$ supports a plurisubharmonic function that is strictly plurisubharmonic everywhere except possibly at a finite number of compact nontrivial subvarieties. Also $(\partial \bar{\partial} \Theta)^{m}=0$ by Proposition 4.1 of $[\mathrm{N}]$. Hence, by the minimal principle of $[\mathrm{B}-\mathrm{T}]$ and $(4.18),\|\Theta\| \equiv 1$. Since $\Theta$ is a holomorphic $(m, 0)$ form, by the Bochner-Weitzenböck formula in Lemma 4.2(a), we have

$$
0=-\Delta_{d}\|\Theta\|^{2} \geq 2\|\nabla \Theta\|^{2} .
$$

Hence, we conclude that $\Theta$ is parallel. This completes the proof of the theorem.

\section{§5. LIOUVILLE THEOREMS FOR PLURISUBHARMONIC FUNCTIONS ON ALE MANIFOLDS}

It was proved by Yau $[\underline{\mathrm{Y}}]$ and Cheng and Yau $[\mathrm{C}-\mathrm{Y}]$ that there is no nonconstant harmonic function with sublinear growth on a complete noncompact manifold with nonnegative Ricci curvature. This is certainly not true for subharmonic functions. However in $\mathbb{C}$, there is no nonconstant subharmonic function that grows slower than $\log r$ where $r$ is the distance function. Hence, this is also true for plurisubharmonic functions on $\mathbb{C}^{m}$. To fix terminologies, let us introduce the following definition. A complete noncompact Kähler manifold $M$ is said to have strong Liouville property for plurisubharmonic functions if there is no nonconstant plurisubharmonic function $\psi(x)$ satisfying

$$
\limsup _{x \rightarrow \infty} \frac{\psi(x)}{\log r(x)}=0 .
$$

We also say that $M$ has Liouville property for plurisubharmonic functions if any bounded plurisubharmonic function on $M$ is constant. All functions are assumed to be smooth in our consideration. In $[\mathbf{N}$, the first author proved that if $M$ is a quasiprojective variety, then $M$ has Liouville property for plurisubharmonic functions. Some other results were also obtained in [N-S-T] on certain Kähler manifolds with nonnegative Ricci curvature.

Notice that the Ricci form of a Kähler manifold with nonnegative (or nonpositive) Ricci curvature is a nonnegative (or nonpositive) real $(1,1)$ form. On the other hand, for a plurisubharmonic function $u, \sqrt{-1} \partial \bar{\partial} u$ is also a nonnegative real $(1,1)$ form. Hence, it is not surprising that the methods in $\S 3$ might be useful in the study of the strong Liouville property for plurisubharmonic functions on ALE Kähler manifolds. This is the purpose of this section. To be consistent with the notation in $\S 4, \Delta$ is again the negative of the usual Beltrami-Laplace operator for functions on manifolds.

We first start with the following lemma.

Lemma 5.1. Let $M^{n}$ be a complete Riemannian ALE manifold of order $\lambda(t)$ satisfying (1.3) and let $o \in M$ be a fixed point. Let $f$ be a nonnegative locally Hölder continuous function on $M$. Suppose the Poisson equation $-\Delta u=f$ has a solution 
$u$ such that

$$
\limsup _{x \rightarrow \infty} \frac{u(x)}{\log r(x)}=0
$$

Then

$$
\int_{0}^{r}\left(\frac{s}{V_{x}(s)} \int_{B_{x}(s)} f(y) d v_{y}\right) d s=o(\log r) .
$$

Proof. First note that it is sufficient to prove (5.2) with $x=o$. Moreover, (5.2) is equivalent to

$$
\int_{r_{0}}^{r}\left(\frac{1}{s^{n-1}} \int_{B_{x}(s)} f(y) d v_{y}\right) d s=o(\log r)
$$

for some $r_{0}$. Lift $u$ and $f$ to be functions in $\mathbb{R}^{2 m} \backslash B_{0}(R)$ and extend $u$ and $f$ to be smooth functions on $\mathbb{R}^{2 m}$, which will also be denoted by $u$ and $f$. Moreover, extend the pullback metric $h$ on $\mathbb{R}^{2 m} \backslash B_{0}(R)$ to $\mathbb{R}^{2 m}$, which will also be denoted by $h$. Then with respect to this metric, $-\Delta u=f$ outside a compact set. Since $h$ is uniformly equivalent to the Euclidean metric, the minimal positive Green's function is equivalent to the minimal positive Green's function of $\mathbb{R}^{2 m}$ with the standard metric [L-S-W p. 67]. We can find a function $\sigma$ which is asymptotically zero at infinity and if $\tilde{u}=u+\sigma$, then $-\Delta \tilde{u}=f$ on $\mathbb{R}^{2 m}$ with $\limsup _{x \rightarrow \infty} \tilde{u}(x) / \log |x|=0$. Here we denote the points on $\mathbb{R}^{2 m}$ by $x$ again. In this set-up, it is sufficient to prove (5.3) on $\mathbb{R}^{2 m}$ with the lifted $f$. Since $f$ is nonnegative outside a compact set and $h$ is uniformly equivalent to the Euclidean metric, one can use Lemma 2.1 together with Remark 1.1 in [T, proceed as in the proof Theorem 2.1 in [N-S-T] and one can prove that the lemma is true. Here one also uses the fact that for fixed $r_{0}$, $\int_{B_{0}\left(r_{0}\right)} G_{R}(0, y) d v_{y}$ is uniformly bounded independent of $R$.

Theorem 5.1. Let $M^{m}$ be a complete ALE Kähler manifold of complex dimension $m$ of order $\eta \geq 2$. Then it has the strong Liouville property for plurisubharmonic functions.

Proof. We may assume that $m \geq 2$, because the case that $m=1$ is obvious. Let $\psi$ be a plurisubharmonic function satisfying (5.1). By Lemma 5.1, we have

$$
\int_{0}^{r}\left(\frac{1}{s^{n-1}} \int_{B_{x}(s)}-\Delta \psi(y) d v_{y}\right) d s \leq o(\log r)
$$

as $r \rightarrow \infty$. Hence one can proceed as in the proof of Theorem 3.1 to conclude that $\partial \bar{\partial} \psi \equiv 0$. We claim that $\psi$ is asymptotically constant at infinity. If this is true, then $\psi$ must be constant by the maximum principle. To prove the claim, we can lift $\psi$ to be a function on $\mathbb{R}^{2 m} \backslash B_{0}(R)$ for some $R$. We can also extend the pullback metric $h$ to the whole $\mathbb{R}^{2 m}$. We also extend $\psi$ to be smooth in $\mathbb{R}^{2 m}$. Since this metric is uniformly equivalent to the Euclidean metric, there is a function $\sigma$ such that $\sigma(x) \rightarrow 0$ as $x \rightarrow \infty$ and $\sigma+\psi$ is harmonic with respect to $h$. Here we denote the points in $\mathbb{R}^{2 m}$ by $x$ again. By Moser's Harnack inequality $\mathrm{Mr}$ and the fact that $\sigma(x)+\psi(x) \leq \log |x|$ for large $|x|$, if $R$ is large, then

$$
\sup _{B_{0}(R)}(\log (2 R)-(\sigma+\psi)) \leq C_{1} \inf _{B_{0}(R)}(\log (2 R)-(\sigma+\psi))
$$


for some constant $C_{1}$ independent of $R$. Hence,

$$
\inf _{B_{0}(R)}(\sigma+\psi) \geq-C_{2}(\log R+1)
$$

for some constant $C_{2}$ independent of $R$. In particular,

$$
\sup _{B_{0}(R)}|\sigma+\psi| \leq C_{3}(\log R+1)
$$

for some constant independent of $R$. By Moser's result again $[\mathrm{Mr}], \sigma+\psi$ must be constant. Since $\sigma(x) \rightarrow 0$ as $x \rightarrow \infty$, we conclude that $\psi$ is asymptotically constant near infinity of $\mathbb{R}^{2 m}$ and hence is asymptotically constant near infinity of $M$. This completes the proof of the theorem.

Combining Theorem 5.1 with Theorem 1.5 of [B-K-N], we have the following result.

Corollary 5.1. Let $M$ be a complete Ricci flat Kähler manifold of complex dimension $m$ with

(a) $V_{o}(r) \geq C r^{2 m}$ for some $o \in M$ and $C>0$,

(b) $\int_{M}|R m|^{m} d v<\infty$, where $R m$ is the curvature tensor of $M$.

Then $M$ has the strong Liouville property for plurisubharmonic functions.

Proof. It is clear that we only need to prove the Corollary for $m>1$. By Theorem 1.5 of $[\mathrm{B}-\mathrm{K}-\mathrm{N}]$, we know that $M$ is an ALE manifold of order $2 m$. The result then follows from Theorem 5.1.

Note that by Corollary 3.1, if $M^{m}$ is a complete noncompact Kähler manifold such that

(a) $|R m|(x) \leq C r^{-2-\epsilon}(x)$ for some constant $C>0$, where $R m$ is the Riemannian curvature tensor;

(b) $V_{o}(r) \geq C r^{2 m}$ for some positive constant $C>0$;

(c) either $\operatorname{Ric}(M) \geq 0$ everywhere or $\operatorname{Ric}(M) \leq 0$ everywhere.

Then $M$ is Ricci flat and hence $M$ has the strong Liouville property for plurisubharmonic functions.

In the proof of Theorem 5.1, one only needs the existence of a smooth plurisubharmonic function $u$ that is strictly plurisubharmonic outside a set of measure zero and satisfies $|\nabla u(x)| \leq C r^{-1}(x)$ and $\sqrt{-1} \partial \bar{\partial} u(x) \leq C r^{-2}(x)$ for some constant $C$, provided the manifold has strong Liouville property for harmonic functions: Every harmonic function $\psi$ on $M$ with $\lim \sup _{x \rightarrow \infty} \psi(x) / \log r(x)=0$ is constant. Hence, by the result of [N-S-T] (see also $[\mathrm{Mk}$ ), we have the following.

Corollary 5.2. Let $M$ be a complete Kähler manifold of complex dimension $m$ with nonnegative bisectional curvature. Suppose that $M$ satisfies

(a) $\operatorname{Ric}_{M}>0$ everywhere,

(b) the scalar curvature $\mathcal{R}$ satisfies $\mathcal{R}(x) \leq \frac{C}{r^{2}(x)}$, for some positive constant $C$, and

(c)

$$
\int_{0}^{\infty} \frac{1}{V_{o}(t)} \int_{B_{o}(t)} \mathcal{R}(y) d v_{y} d t<\infty .
$$

Then $M$ has strong Liouville property for plurisubharmonic functions.

Proof. By [N-S-T], a strictly plurisubharmonic function $u$ satisfying the above mentioned properties can be constructed by solving the Poisson equation on $M$ (cf. 
Theorem 5.1 and Corollary 1.2 in [N-S-T] $)$. By the gradient estimate of [C-Y], $M$ also has the strong Liouville property for harmonic functions. Using Theorem 2.1 of $[\mathrm{N}-\mathrm{S}-\mathrm{T}]$ we also have

$$
\int_{0}^{r} \frac{t}{V_{o}(t)} \int_{B_{o}(t)}-\Delta \psi d v_{y} d t=o(\log r)
$$

The result then follows by the argument in the proof of Theorem 3.1.

\section{REFERENCES}

[Ab] U. Abresch, Lower curvature bounds, Toponogov's theorem, and bounded topology, Ann. Sci. École Norm. Sup. 18 (1985), 651-670. MR 87j:53058

[An] M.-T. Anderson, The compactification of a minimal submanifold in Euclidean spaces by the Gauss map, IHES preprint, 1984.

[B-K-N] S. Bando, A. Kasue and H. Nakajima, On a construction of coordinates at infinity on manifolds with faster curvature decay and maximum volume growth, Invent. Math. 97 (1989), 313-349. MR 90c:53098

[Ba] R. Bartnik, The mass of an asymptotically flat manifold, Comm. Pure Appl. Math. 34 (1986), 661-693. MR 88b:58144

[Be] A. Besse, Einstein Manifolds, Springer-Verlag, Berlin, 1987. MR 88f:53087

[B-T] E. Bedford and B. A. Taylor, The Dirichlet problem for a complex Monge-Ampère equation, Invent. Math. 37 (1976), 1-44. MR 56:3351

[C] E. Calabi, Métriques kählériennes et fibrés holomorphes, Ann. Sci. École Norm. Sup. 12 (1979), 269-294. MR 83m:32033

[C-G] J. Cheeger and D. Gromoll, The splitting theorem for manifolds of nonnegative Ricci curvature, J. Diff. Geom. 6 (1971/72), 119-128. MR 46:2597

[C-Z] B.-L. Chen and X.-P. Zhu, On complete Kähler manifolds with positive bisectional curvature, preprint.

[C-Y] S. Y. Cheng and S.-T. Yau, Differential equations on Riemannian manifolds and their geometric applications, Comm. Pure Appl. Math. 28 (1975), 333-354. MR 52:6608

[DR] G. de Rham, Variétés différentiables, Hermann, Paris, 1955. MR 16:957b

[Ds] G. Drees, Asymptotically flat manifolds of nonnegative curvature, Differential Geom. Appl. 4 (1994), 77-90. MR 95e:53052

[E-H] T. Eguchi and A. J. Hanson, Asymptotically flat self-dual solutions to Euclidean gravity, Ann. Physics 120 (1979), 82-106. MR 80h:83011

[G-H] G.-W Gibbons and S. Hawking, Gravitational multi-instantons, Phys. Lett. B78 (1978), 430-432.

[Gt] H. Grauert, Über Modifikationen und exzeptionelle analytische Mengen, Math. Ann. 146 (1962), 331-368. MR 25:583

[Ge] R. E. Greene, A genealogy of noncompact manifolds of nonnegative curvature: history and logic, Comparison geometry (1997), 99-134.

[G-P-Z] R. E. Greene, P. Petersen and S.-H. Zhu, Riemannian manifolds of faster-than-quadratic curvature decay. Internat. Math. Res. Notices no. 9, 1994, pp. 363-377. MR 95m:53054

[G-W 1] R. E. Greene and H. Wu, Function theory on manifolds which possess a pole, Lecture Notes in Mathematics, vol. 699, Springer, Berlin, 1979. MR 81a:53002

[G-W 2] R. E. Greene and $\mathrm{H}$. Wu, $C^{\infty}$ approximations of convex, subharmonic, and plurisubharmonic functions, Ann. Sci. École Norm. Sup. 12 (1979), 47-84. MR 80m:53055

[G-W 3] R. E. Greene and H. Wu, Gap theorems for noncompact Riemannian manifolds, Duke Math. J. 49 (1982), 731-756. MR 84m:53050

[G-R] R. Gunning and H. Rossi, Analytic Functions of Several Complex Variables, PrenticeHall, Englewood Cliffs, N.J., 1965. MR 31:4927

$[\mathrm{H}] \quad$ N. Hitchin, Polygons and gravitons, Math. Proc. Cambridge Philos. Soc. 85 (1979), 465-476. MR 80h:53047

[J] D. Joyce, Asymptotically locally Euclidean metrics with holonomy $S U(m)$, Annals of Global Analysis and Geometry 19 (2000), 55-73. MR 2002i:53063

[Ka] A. Kasue, Gap theorems for minimal submanifolds of Euclidean space, J. Math. Soc. Japan 38 (1986), 473-492. MR 87h:53084 
[K-S] A. Kasue and K. Sugahara, Gap theorems for certain submanifolds of Euclidean spaces and hyperbolic space forms, Osaka J. Math. 24 (1987), 679-704. MR 89a:53072

[Kr] P. Kronheimer, The construction of ALE spaces as hyper-Kähler quotients, J. Diff. Geom. 29 (1989), 665-683. MR 90d:53055

[L-P] J. Lee and T. Parker, The Yamabe problem, Bull. Amer. Math. Soc. 17 (1987), 37-91. MR 88f:53001

[L-S] P. Li and R. Schoen, $L^{p}$ and mean value properties of subharmonic functions on Riemannian manifolds, Acta Math. 153 (1984), 279-301. MR 86j:58147

[L-Y] P. Li and S.-T. Yau, On the parabolic kernel of the Schrödinger operator, Acta Math. 156 (1986), 153-201. MR 87f:58156

[L-S-W] W. Littman, G. Stampacchia and H. F. Weinberger, Regular points for elliptic equations with discontinuous coefficients, Ann. Scuola Norm. Sup. Pisa 17 (1963), 43-77. MR 28:4228

[Mk] N. Mok, An embedding theorem of complete Kähler manifolds of positive bisectional curvature onto affine algebraic varieties, Bull. Soc. Math. France 112 (1984), 197-250. MR 87a:53103

[Me] H. Moore, Minimal submanifolds with finite total scalar curvature, Indiana Univ. Math. Jour. 45 (1996), 1021-1043. MR 98e:58053

[My] C. Morrey, Multiple Integrals in Calculus of Variations, Springer-Verlag, New York, 1966. MR 34:2380

[Mr] J. Moser, On Harnack's theorem for elliptic differential equations, Comm. Pure Appl. Math. 14 (1961), 577-591. MR 28:2356

[M-S] J.-H. Michael and L. Simon, Sobolev and mean-value inequalities on generalized submanifolds of $R^{n}$, Comm. Pure Appl. Math. 26 (1973), 361-379. MR 49:9717

[M-S-Y] N. Mok, Y.-T. Siu and S.-T. Yau, The Poincaré-Lelong equation on complete Kähler manifolds, Compositio Math. 44 (1981), 183-218. MR 84g:32011

[M-K] J. A. Morrow and K. Kodaira, Complex manifolds, Holt, Rinehart and Winston, New York, 1971. MR 46:2080

[Na] R. Narasimhan, The Levi problem for complex spaces, Math. Ann. 142 (1960/1961), 335-365. MR 26:6439

[N] L. Ni, Vanishing theorems on complete Kähler manifolds and their applications, J. Differential Geom. 50 (1998), 89-122. MR 2000c:32069

[N-S-T] L. Ni, Y.-G. Shi and L.-F. Tam, Poisson equation, Poincaré-Lelong equation and curvature decay on complete Kähler manifolds, J. Diff. Geom. 57 (2001), 339-388. MR 2002j:53042

[S] W.-X. Shi, Ricci flow and the uniformization on complete noncompact Kähler manifolds. J. Differential Geom. 45 (1997), 94-220. MR 98d:53099

[Sc-Y] R. Schoen and S.-T. Yau, Proof of the positive mass theorem, II, Comm. Math. Phys. 79 (1981), 231-260. MR 83i:83045

[Si-Y] Y.-T. Siu and S.-T. Yau, Complete Kähler manifolds with nonpositive curvature faster than quadratic decay, Annals of Math. 105 (1977), 225-264. MR 55:10719

[T] L.-F . Tam, Liouville properties of harmonic maps, Math. Res. Lett. 2 (1995), 719-735. MR 96k:58063

[T-Y] G. Tian and S.-T. Yau, Complete Kähler manifolds with zero Ricci curvature II, Invent. Math. 106 (1991), 27-60. MR 92j:32028

[Y] S.-T. Yau, Harmonic functions on complete Riemannian manifolds, Comm. Pure Appl. Math. 28 (1975), 201-228. MR 55:4042

[Yg] S.-K. Yeung, Complete Kähler manifolds of positive Ricci curvature, Math. Z. 204 (1990), 187-208. MR 91h:53065

Department of Mathematics, University of California, San Diego, La Jolla, CaliFORNIA 92093

E-mail address: lni@math.ucsd.edu

Department of Mathematics, Peking University, Beijing, 100871, China

E-mail address: ygshi@math.pku.edu.cn

Department of Mathematics, The Chinese University of Hong Kong, Shatin, Hong Kong, China

E-mail address: lftam@math.cuhk.edu.hk 